\title{
Transcriptional targeting of oncogene addiction in medullary thyroid cancer
}

\author{
Anisley Valenciaga, ${ }^{1}$ Motoyasu Saji, ${ }^{1}$ Lianbo Yu, ${ }^{2}$ Xiaoli Zhang, ${ }^{2}$ Ceimoani Bumrah, ${ }^{3}$ Ayse S. Yilmaz, ${ }^{4}$ \\ Christina M. Knippler, ${ }^{1}$ Wayne Miles, ${ }^{5}$ Thomas J. Giordano, ${ }^{6}$ Gilbert J. Cote, ${ }^{7}$ and Matthew D. Ringel' \\ 'Division of Endocrinology, Diabetes, and Metabolism, The Ohio State University Wexner Medical Center and Arthur \\ G. James Comprehensive Cancer Center, Columbus, Ohio, USA. ${ }^{2}$ Center for Biostatistics, ${ }^{3}$ College of Arts and Sciences, \\ ${ }^{4}$ Department of Biomedical Informatics, and ${ }^{5}$ Department of Molecular Genetics, The Ohio State University, Columbus, \\ Ohio, USA. ${ }^{6}$ Department of Pathology, University of Michigan, Ann Arbor, Michigan, USA. 'Department of Endocrine \\ Neoplasia and Hormonal Disorders, University of Texas MD Anderson Cancer Center, Houston, Texas, USA.
}

\begin{abstract}
Metastatic medullary thyroid cancer (MTC) is incurable and FDA-approved kinase inhibitors that include oncogenic RET as a target do not result in complete responses. Association studies of human MTCs and murine models suggest that the CDK/RB pathway may be an alternative target. The objective of this study was to determine if CDKs represent therapeutic targets for MTC and to define mechanisms of activity. Using human MTC cells that are either sensitive or resistant to vandetanib, we demonstrate that palbociclib (CDK4/6 inhibitor) is not cytotoxic to MTC cells but that they are highly sensitive to dinaciclib (CDK1/2/5/9 inhibitor) accompanied by reduced CDK9 and RET protein and mRNA levels. CDK9 protein was highly expressed in 83 of 83 human MTCs and array-comparative genomic hybridization had copy number gain in 11 of 30 tumors. RNA sequencing demonstrated that RNA polymerase II-dependent transcription was markedly reduced by dinaciclib. The CDK7 inhibitor THZ1 also demonstrated high potency and reduced RET and CDK9 levels. ChIP-sequencing using H3K27Ac antibody identified a superenhancer in intron 1 of RET. Finally, combined inhibition of dinaciclib with a RET kinase inhibitor was synergistic. In summary, we have identified what we believe is a novel mechanism of RET transcription regulation that potentially can be exploited to improve RET therapeutic targeting.
\end{abstract}

Conflict of interest: The authors have declared that no conflict of interest exists.

Submitted: May 14, 2018 Accepted: July 10, 2018 Published: August 23, 2018

Reference information: JCI Insight. 2018;3(16):e122225. https://doi.org/10.1172/jici. insight.122225.

\section{Introduction}

Medullary thyroid cancer (MTC) is an aggressive neuroendocrine form of thyroid cancer that represents $1 \%-2 \%$ of all thyroid cancer cases in the United States, but disproportionally accounts for $14 \%$ of all thyroid cancer-related deaths $(1,2)$. Patients presenting with MTC localized to the thyroid have a 10-year survival rate of $95 \%$ (3). However, approximately $70 \%$ of patients present with lymph node metastases and approximately $10 \%$ with distant metastases at the time of diagnosis (4). Metastatic MTC carries a 10 -year survival rate of 21\% (5). MTC arises from calcitonin-secreting parafollicular C-cells of the thyroid. These cells do not process iodine, making traditional radioactive iodine treatment ineffective for progressive disease cases. Currently, there are 2 FDA-approved therapies for metastatic MTC, vandetanib and cabozantinib, both of which are multikinase inhibitors (MKIs) that include the rearranged during transfection (RET) tyrosine kinase, the most common driver mutation in MTC, as a target (6, 7). Although progression-free survival is lengthened by these 2 compounds, the effect is transient, associated with side effects, complete responses have not been attained, and long-term survival improvements have not been reported $(6,8,9)$. Thus, new treatments are needed for both initial therapy and for patients with treatment resistance or who do not tolerate these 2 agents.

MTC can present as a hereditary disease in $25 \%$ of cases as a phenotype of multiple endocrine neoplasia type II (MEN2) syndromes A and B, or as a sporadic disease in $75 \%$ of cases (10). Constitutively activating mutations in RET cause almost all hereditary occurrences and approximately $50 \%$ of sporadic cases (11); thus, this protein has been the primary focus of drug development. Activated RET signals through a number of downstream pathways including PI3K/AKT and MEK/ERK. In addition to activating mutations in $R E T$, somatic mutations in $R A S$ genes (most frequently $H-R A S$ ) also have been described in MTC, albeit at a lower frequency than $R E T$ mutations in most populations (12-14). Somatic RET mutations in nonfamilial MTC 
cases are nearly all at codon 918. In contrast, a variety of germline RET mutations can cause MEN2, with the 918 mutation being limited to the most aggressive form of the disease, MEN2B (11). Interestingly, murine models consistently have shown that heterozygous generalized loss of retinoblastoma (RB) and other members of the RB pathway including cyclin-dependent kinase (CDK) inhibitors p18 and p27, unexpectedly cause MTC and have been shown to cooperate with Ret mutants in vivo (15-19). Also, it has been reported that murine models with CDK5 overactivation developed MTC in an RB-mediated mechanism (20). These data suggest a potentially unique sensitivity of neuroendocrine thyroid cells to alterations in CDK/RB pathway signaling. In human MTCs, we, and others, have identified loss of heterozygosity (LOH) at the p18 and E2F2 loci, and we recently demonstrated that loss of RB is associated with reduced MTC disease-specific survival $(16,21-23)$. However, there have not been reports of germline $R B$ mutations in patients diagnosed with MTC, although the thyroid glands have not been systematically evaluated to our knowledge.

In addition to regulating the cell cycle, CDKs, such as CDK7 and CDK9, play an essential role in transcription regulation and have been targeted in cancer. Gene transcription is exquisitely regulated through a variety of mechanisms, particularly at the initiation and elongation steps of RNA polymerase II (RNAP II) (24-26). During transcription initiation, the preinitiation complex is assembled, followed by the phosphorylation of the carboxy-terminal domain (CTD) of RNAP II at Ser5 by CDK7 (27). RNAP II then proceeds to an intrinsic pause site, where it is stalled by the negative elongation factor (NELF) and DRB sensitivity-inducing factor (DSIF) $(26,28)$. Transcription elongation requires release from this promoterproximal pause site. This occurs by phosphorylation of DSIF, NELF, and the CTD of RNAP II at Ser2 by $\mathrm{CDK}$, the catalytic subunit of the positive transcription elongation factor $\mathrm{b}$ (P-TEFb). P-TEFb is recruited to the RNAP II complex to allow for this activity by bromodomain-containing proteins (e.g., BRD4), CDK8, $\mathrm{NF}-\mathrm{\kappa B}$, and DNA-binding transcription factors. BRD4 has been specifically targeted with therapeutic intent and is a member of the bromodomain and extraterminal domain (BET) family of chromatin readers that includes BRD4, BRD2, BRD3, and BRDT (29). These proteins contain 2 bromodomains that bind acetylated lysine residues in histone and nonhistone proteins, facilitating the anchoring of nuclear macromolecular complexes such as HATs, histone deacetylases, and Mediator complexes (30, 31).

Preclinical models using CDK7 inhibitors show antiproliferative and proapoptotic effects against difficult-to-treat solid tumors including triple-negative breast, small-cell lung, and ovarian cancers (32-34). In addition, a number of CDK inhibitors that include CDK9 targeting in their profile have been shown to decrease phosphorylation of RNAP II and induce apoptosis by inhibiting transcription of antiapoptotic proteins such as XIAP and Bcl-2 family proteins (35-42). BRD4 inhibitors that displace the BET bromodomain from chromatin also have progressed in preclinical models. In thyroid cancer, mouse anaplastic thyroid cancer models are sensitive to BRD4 inhibition and show reduced tumor burden and suppression of MYC expression (43-45).

Superenhancers have been identified as regulators of key genes involved in cellular fate and oncogenesis. Superenhancers are clusters of enhancers and DNA regulatory elements that are densely occupied by transcription factors, cofactors, and chromatin regulators. They facilitate high expression of genes that define cell identity in healthy and tumor cells; the genes that they regulate are especially sensitive to perturbations in enhancer function $(46,47)$. Identification of superenhancers is aided by enrichment of H3K27Ac chromatin mark on these areas along with high occupancy of BRD4 and Mediator complexes (48, 49). CDK7 is also an important positive regulator of superenhancer-mediated transcription, and expression of superenhancer-associated genes has been shown to be particularly vulnerable to transcriptional inhibitors that target CDK7 and BRD4 $(26,46,50,51)$.

In the present study, we determined that human MTC cells are relatively resistant to CDK4/6 inhibition but were specifically sensitive to CDK7 and/or CDK9 inhibition in coordination with loss of RET mRNA. Subsequently, we identify, for the first time to our knowledge, the presence of a superenhancer in intron 1 of $R E T$ and provide evidence for transcriptional RET targeting both as monotherapy and in combination with RET kinase inhibitors. Taken together, these data suggest that transcriptional targeting of $R E T$ may represent a novel therapeutic approach.

\section{Results}

$C D K 4 / 6$ inhibitor reduces $M T C$ cell proliferation but does not affect cell viability in vitro. Due to the sensitivity of MTC to RB loss and the clinical data associating loss of RB function with aggressive MTC (21), palbociclib, a relatively specific CDK4/6 inhibitor, was used initially to target the CDK/RB pathway in MTC cells 
in vitro. Cell viability assays showed a statistically significant reduction in TT cell viability at 96 hours of exposure using $10 \mu \mathrm{M}$ palbociclib, and at 120 hours of exposure using $5 \mu \mathrm{M}$ and $10 \mu \mathrm{M}$ concentrations (Supplemental Figure 1A; supplemental material available online with this article; https://doi.org/10.1172/ jci.insight.122225DS1). However, $\mathrm{IC}_{50}$ values were not reached in the assays. Concordantly, cell viability was not reduced at any of the time points tested in MZ-CRC-1 cells. Cell counting assays demonstrated cell growth inhibition at long exposure times (Supplemental Figure 1B). TT cell growth was reduced with $1 \mu \mathrm{M}$ and $10 \mu \mathrm{M}$ palbociclib exposure for 72-120 hours, while MZ-CRC-1 cell number was significantly reduced at $10 \mu \mathrm{M}$ for $72-120$ hours of exposure. $\mathrm{GI}_{50}$ for the TT cell line at 72 hours of exposure was $1.8 \mu \mathrm{M}(95 \%$ CI: $0.7-5.2 \mu \mathrm{M})$ and $0.3 \mu \mathrm{M}(95 \% \mathrm{CI}: 0.2-0.6 \mu \mathrm{M})$ at 96 hours. In the MZ-CRC-1 cell line, the effect was more modest, with $\mathrm{GI}_{50}$ not reached at 72 hours in these assays, and having a value of $12.3 \mu \mathrm{M}(95 \% \mathrm{CI}$ : $2.8-166.3 \mu \mathrm{M})$ at 96 hours and $4.9 \mu \mathrm{M}(95 \% \mathrm{CI}$ : $1.7-14.0 \mu \mathrm{M})$ at 120 hours of exposure to palbociclib. Taken together, the data suggest that both cell lines were relatively resistant to palbociclib.

CDK1/2/5/9 inhibitor dinaciclib is active against naive and vandetanib-resistant MTC cells in vitro. To expand our analysis of CDK targeting in these cells, including some implicated in MTC development (20), we next tested the effects of the FDA-approved multi-CDK inhibitor dinaciclib, which inhibits primarily CDK1, $-2,-5$, and -9 activities. In contrast to palbociclib, dinaciclib resulted in reduced cell viability in a doseand time-dependent manner at low concentrations in both MTC cell lines (Figure $1 \mathrm{~A}) . \mathrm{IC}_{50}$ values were achieved starting at 72 hours of exposure in this assay for both cell lines; at 72 hours, $\mathrm{IC}_{50}$ was $4.6 \mathrm{nM}(95 \%$ CI: 3.2-6.4 nM) for the TT cell line and $13.9 \mathrm{nM}(95 \% \mathrm{CI}: 10.9-19.2 \mathrm{nM})$ for the MZ-CRC-1 line. Cell proliferation was also reduced by dinaciclib in a time- and dose-dependent manner at low concentrations (Figure 1B). $\mathrm{GI}_{50}$ concentrations were achieved starting at 24 hours for TT and 48 hours for MZ-CRC-1; after 72 hours of exposure to dinaciclib, $\mathrm{GI}_{50}$ was $7.8 \mathrm{nM}(95 \% \mathrm{CI}$ : $3.6-5.3 \mathrm{nM})$ for TT cells and $3.8 \mathrm{nM}$ (95\% CI: 3.0-5.0 nM) for MZ-CRC-1 cells.

Given FDA-approval of vandetanib as a first-line therapy for patients with progressive metastatic MTC cases, we tested dinaciclib on MTC cells that have been cultured in vandetanib-containing medium and selected for vandetanib resistance, along with age-matched controls. The resistant cells were also sensitive to dinaciclib (Figure 2, A and B). For the TT cells, $\mathrm{IC}_{50}$ concentrations were reached starting at 48 hours for vandetanib-resistant and age-matched controls. At 72 hours, the $\mathrm{IC}_{50}$ was $4.4 \mathrm{nM}$ (95\% CI: $\left.0.4-16.3 \mathrm{nM}\right)$ for vandetanib-resistant cells and $1.8 \mathrm{nM}$ (95\% CI: 0.54-5.7 nM) for age-matched controls. For MZ-CRC-1 cells, $\mathrm{IC}_{50}$ values were achieved starting at 48 hours for the vandetanib-resistant cells and starting at 72 hours for age-matched controls. At 72 hours, the $\mathrm{IC}_{50}$ was $10.1 \mathrm{nM}$ (95\% CI: 9.3-11.0 nM) for vandetanib-resistant cells and $17.1 \mathrm{nM}(95 \% \mathrm{CI}: 15.3-19.6 \mathrm{nM})$ for the age-matched controls. In addition, trend analysis demonstrated no statistical difference in cell viability between age-matched controls and vandetanib-resistant TT cells, and although some MZ-CRC-1 trends were statistically significant, the $\mathrm{IC}_{50}$ values were similar between age-matched controls and vandetanib-resistant cells, consistent with high activity in both cell lines (Supplemental Figures 2 and 3).

Dinaciclib treatment induces apoptosis in MTC cells in vitro. To determine a cause of cell death induced by dinaciclib, Western blots were performed to detect PARP cleavage. These experiments identified PARP-1 cleavage in both cell lines (Figure 3). At 72 hours of exposure, PARP-1 cleavage in the TT cell line was more pronounced at $10 \mathrm{nM}$ dinaciclib (Figure 3A), matching the cell viability data (Figure 1A). However, at later time points, PARP-1 cleavage became more stable across treatments, coinciding with a similar pattern of diminished cell viability at all concentrations tested, suggesting a maximal effect (Figure $3 \mathrm{~B}$ and Figure 1A). In the case of MZ-CRC-1 cells, PARP-1 cleavage was present starting at $10 \mathrm{nM}$, and total PARP-1 protein expression was highest at $20 \mathrm{nM}$ after 72 hours of dinaciclib treatment, a concentration that reduces MZ-CRC-1 viability by $80 \%$ (Figure $3 \mathrm{~A}$ and Figure $1 \mathrm{~A}$ ).

Dinaciclib treatment downregulates transcription at low concentrations in MTC cells. Due to the multiple targets of dinaciclib, we sought to identify key pathways inhibited by the compound to explore the mechanism of action in an unbiased manner. mRNA sequencing (RNA-seq) of TT and MZ-CRC-1 cells treated with $10 \mathrm{nM}$ and $20 \mathrm{nM}$ dinaciclib, respectively, or vehicle control for 12 hours and 24 hours was performed and identified a global reduction in mRNA levels (Figure 4 and Supplemental Figure 4). Gene score enrichment analysis (GSEA) of the differentially expressed genes revealed that in TT cells, the transcripts involved in RNAP IIregulated transcription were particularly decreased with dinaciclib treatment. In contrast, genes modulated by other RNA polymerase complexes including mitochondrial genes were upregulated and/or unchanged. Many of the downregulated transcripts after dinaciclib treatment are involved in key cellular processes including cell 
A

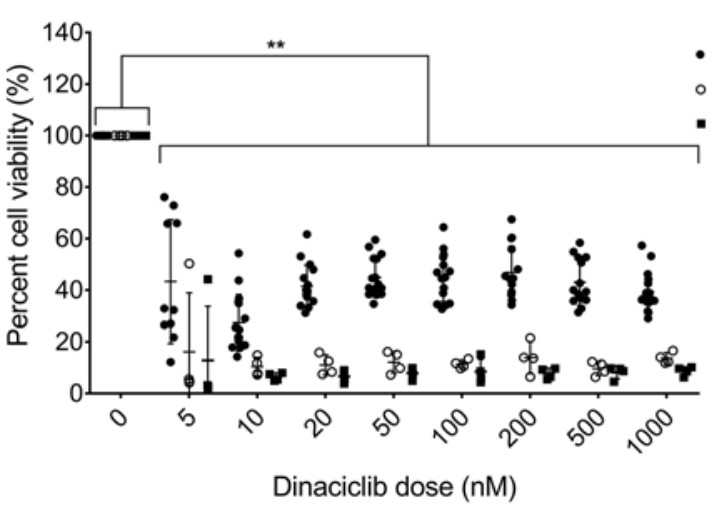

B

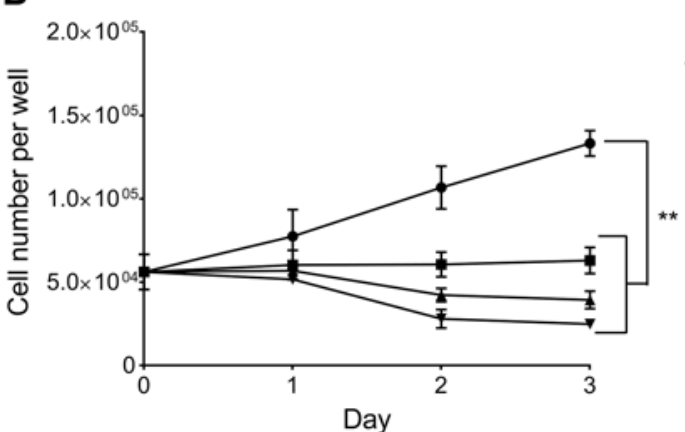

$72 \mathrm{~h} n=16$

- $96 h n=4$

- $120 h n=4$

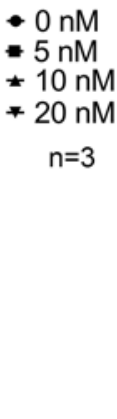

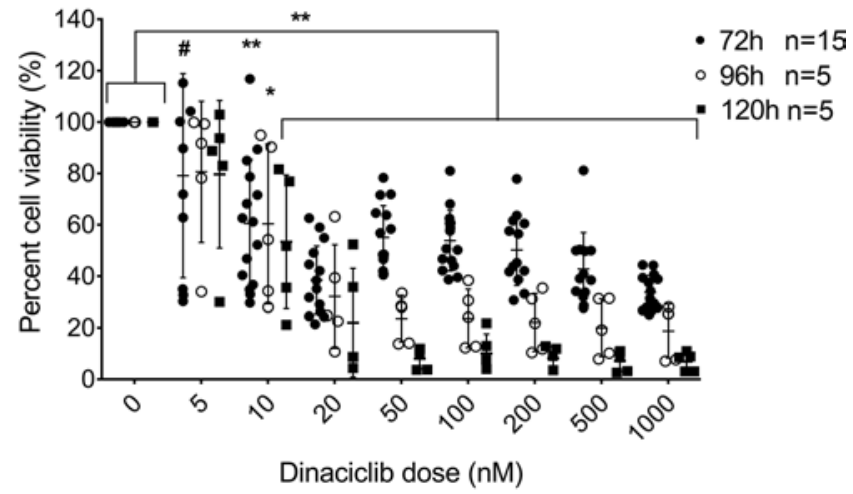

MZ-CRC-1

MZ-CRC-1

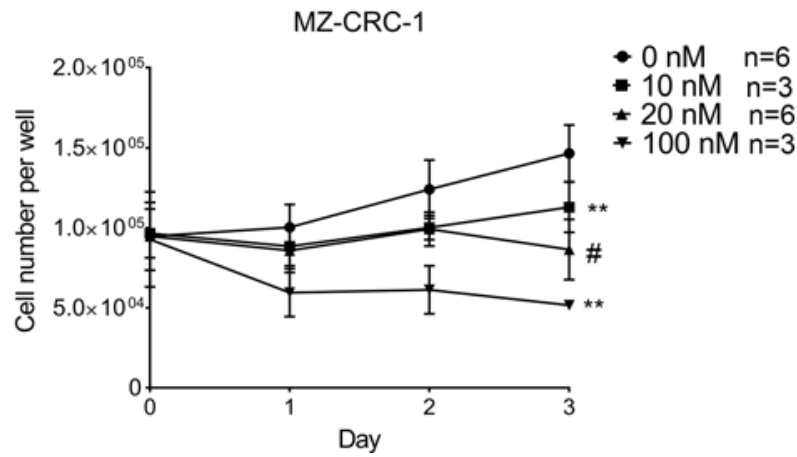

Figure 1. TT and MZ-CRC-1 cell viability and proliferation after dinaciclib treatment. Cell viability assays were performed with TT and MZ-CRC-1 cells treated with dinaciclib for 72-120 hours (A). Data were normalized to $0 \mathrm{nM}$ (vehicle control) wells and represented as percentages. (B) Cell counting assays of TT and MZ-CRC-1 cells treated with dinaciclib were seeded and counted for 3 days after treatment, including vehicle control wells at day 0 . Each biological replicate was performed in triplicate (cell viability assays) or duplicate (cell counting assays), and the error bars represent the standard deviation. Twoway ANOVA tests followed by Holm's procedure were done on both types of assays. In the case of cell counting assays, $\log _{2}$-transformed data were used for statistical analysis. ${ }^{* *} P<0.0001,{ }^{*} P<0.001,{ }^{*} P<0.01$.

cycle transition (CDK6, CCNA1, CCNB1, CCNE1, and CCNG2), chromosome segregation during cell division (CENPA, CENPF, AURKA, and CDC25A), and DNA replication (ORC). In addition, there was enrichment of transcripts related to RNAP I, RNAP III, and mitochondrial polymerase transcription in the treatment group (Figure 4A and Supplemental Table 1). In these gene sets, multiple HIST, RPL, and RPS transcripts were upregulated. Gene enrichment analysis for differentially expressed transcripts in the MZ-CRC-1 cell line elucidated downregulation in the treatment group of transcripts involved in cell cycle transition including DNA replication and DNA repair steps such as ORC, PSM, MCM, POLA2, POLD3, RB1, CDC25A, CDC6, p21, p27, CDK1, CDK4, WEE1, CCNH, CCNA, CCNE, CCND, CENPA, CENPN, CENPQ, and CENPL, among others. Similar to the TT cell line, there was upregulation of HIST and RPL transcripts that are involved in RNAP I, RNAP III, and mitochondrial polymerase-related transcripts in treated MZ-CRC-1 cells (Figure 4B and Supplemental Table 2). A list of the top downregulated and upregulated pathways after dinaciclib treatment are included in Supplemental Tables 3-6. Leading edge analysis of GSEA gene sets was performed, and we identified the pathways that shared multiple genes. These genes were used as input for Gene Ontology biological process classification through the STRING database. As can be seen in Supplemental Tables 7-10, the genes downregulated in treated cells were most typically involved in transcription regulation, nucleic acid metabolic processes, and cell cycle, among other classifications. The genes upregulated in the treated groups were mainly involved in chromatin modification.

Dinaciclib treatment downregulates CDK9 and RET in MTC cells in vitro. When testing protein levels of dinaciclib targets at doses close to the $\mathrm{IC}_{50}$, it was found that levels of $\mathrm{CDK} 1,-2$, and -5 were stable; in contrast, CDK9 protein levels were markedly reduced at low doses (Figure 5A). In support of reduced CDK9 activity, and consistent with the RNA-seq data, we detected lower RNAP II Ser2 phosphorylation, a specific CDK9 phosphorylation site, along with downstream proteins cyclin D1 and Mcl-1, which have short 
A

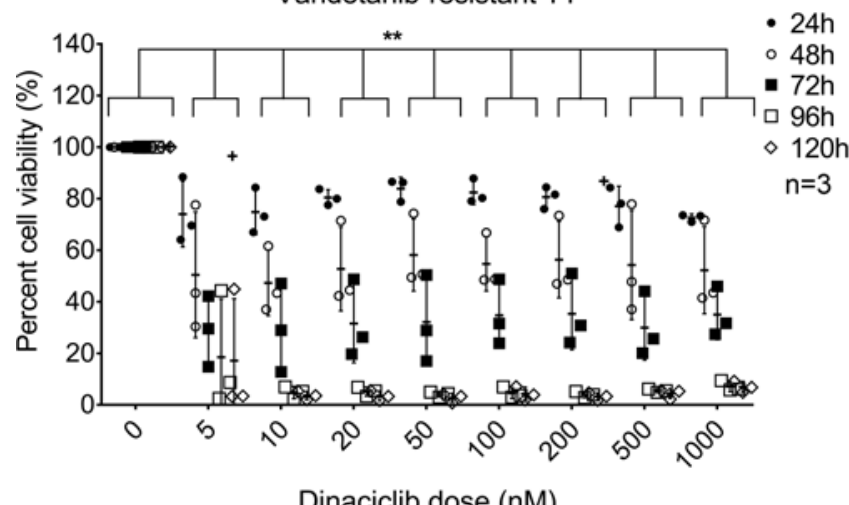

Dinaciclib dose $(\mathrm{nM})$

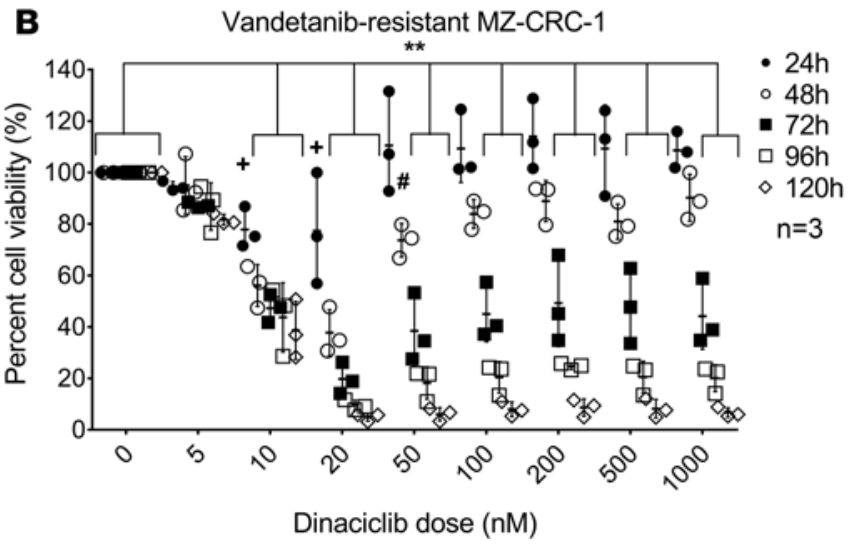

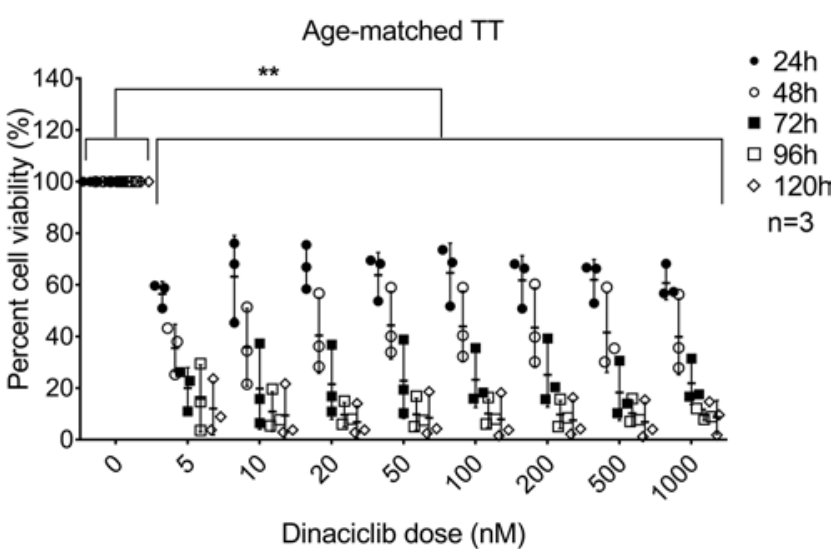

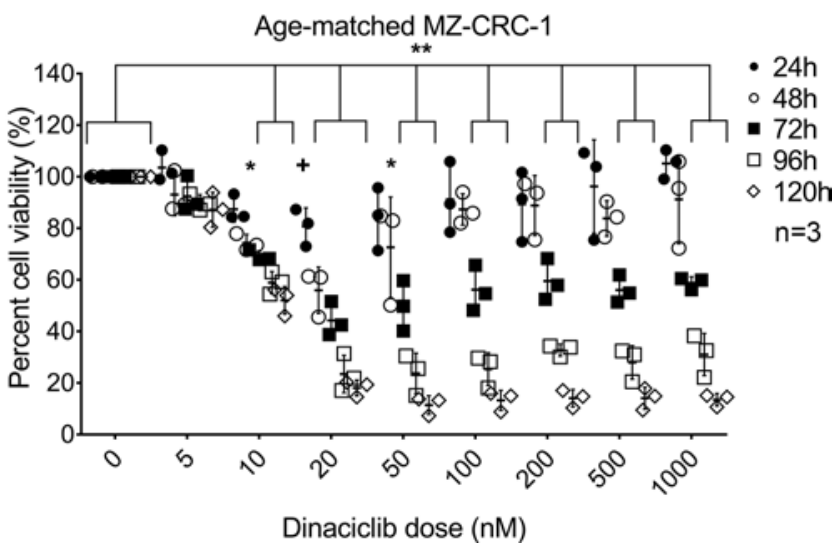

Figure 2. TT and MZ-CRC-1 vandetanib-resistant and age-match control cell viability after dinaciclib treatment. Cell viability assays were performed with TT vandetanib-resistant and age-matched controls (A), and MZ-CRC-1 vandetanib-resistant and age-matched controls (B) treated with dinaciclib for 72-120 hours. The data were normalized to $0 \mathrm{nM}$ (vehicle control) wells and represented as percentages. Each biological replicate was done in triplicate and the error bars represent the standard deviation. Two-way ANOVA tests followed by Holm's procedure were done for statistical analysis. ${ }^{*} P<0.0001,{ }^{*} P<0.001,{ }^{\#} P<0.01,{ }^{+} P<0.05$.

half-lives and are affected by disruption in RNAP II function (Figure 5B). Additionally, CDK9 mRNA levels were reduced in both cell lines at low concentrations and early time points of exposure (Figure 5C), as was also seen in the RNA-seq data. Relative expression versus vehicle control was 0.286 for TT cells at 12 hours $(P=0.0069)$ and 0.132 at 24 hours $(P=0.0087)$. For MZ-CRC-1 cells, the relative expression normalized to control was 0.218 at 12 hours $(P=0.0262)$ and 0.201 at 24 hours $(P=0.0003)$.

RNA-seq also demonstrated a reduction in RET mRNA levels with dinaciclib treatment in both cell lines. This result was confirmed in the MZ-CRC-1 cell line, and a consistent trend was noted in the TT cell line at 24 hours (Figure 6B). RET protein expression also was decreased in both cell lines to a lesser degree, with some increase in the upper band of the RET doublet suggesting posttranslational changes (Figure 6A).

To determine if the changes in RET and CDK9 protein levels related to changes in protein synthesis or stability, we treated both MTC cell lines with dinaciclib in the absence or presence of cycloheximide or bortezomib (Supplemental Figure 5). CDK9 protein levels were not changed with the addition of cycloheximide even though the compound was active as evidenced by the reduction in cyclin D1, a protein with a short halflife. Similarly, exposure to bortezomib did not alter CDK9 levels despite the noted increase in Mcl-1 protein levels, a positive control for this assay. Thus, the reduction of CDK9 levels by dinaciclib are due primarily to transcription or mRNA stability effects. In contrast, cycloheximide treatment alone decreased RET protein levels, indicating a decrease in protein stability with this treatment, but the decrease was more pronounced than using dinaciclib alone at the time points tested. RET protein level did not increase with bortezomib treatment. Thus, RET is regulated both at the mRNA level and by posttranslational mechanisms.

Interestingly, we also noted elevated levels of RET and ERK phosphorylation, consistent with overactivation in conjunction with the increase in the upper band of the RET doublet (Figure 6B). We interpret 

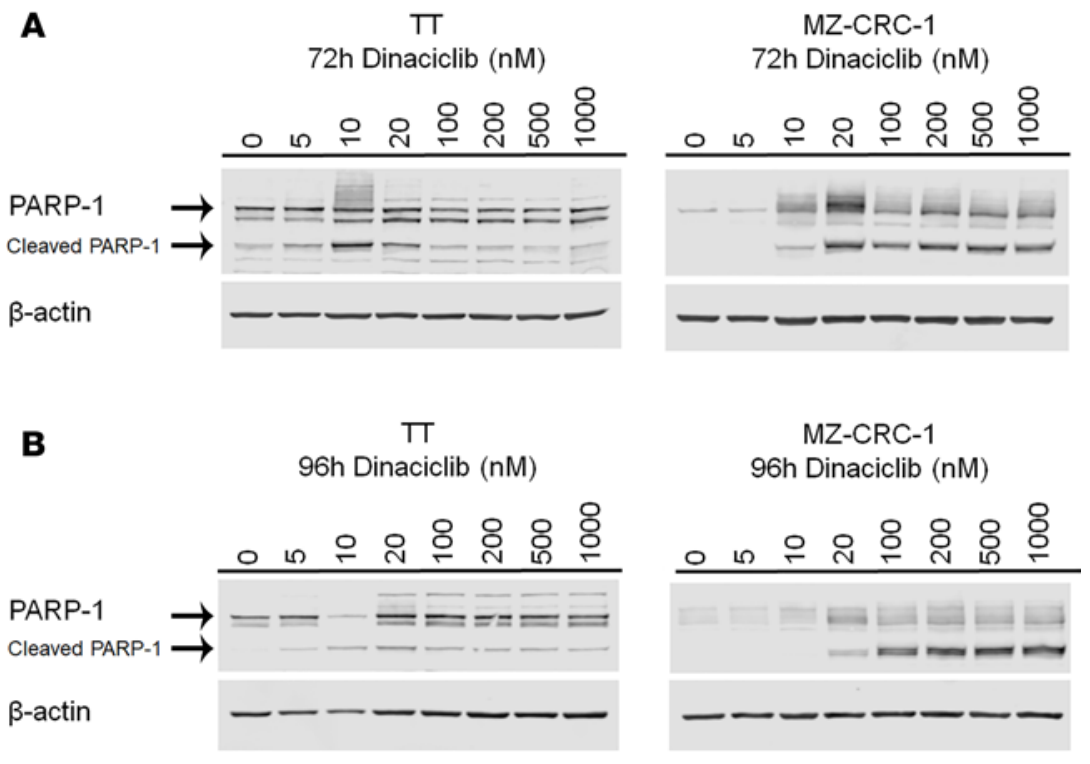

Figure 3. Apoptosis after dinaciclib treatment of TT and MZ-CRC-1 cells. Western blots were performed with protein extracts from TT and MZ-CRC-1 cells treated with vehicle control or dinaciclib for 72 hours (A) and 96 hours (B). PARP-1 cleavage was used as marker of apoptosis and observed in the treated cells versus vehicle control.

these data as consistent with hyperactivation of residual RET protein in response to dinaciclib treatment to enable cell survival, or selection of the subpopulation of MTC cells has the highest level of RET activity.

RET inhibition reduces RET activation after dinaciclib treatment and synergizes with dinaciclib. Because dinaciclib reduced MTC cell viability and proliferation, but increased RET activation, we hypothesized that combining dinaciclib with RET kinase inhibition would be synergistic. To test this hypothesis, we treated TT cells with dinaciclib for 6 hours followed by vandetanib for 72 hours. Western blot showed that the RET and ERK activation that occur with dinaciclib treatment were inhibited by vandetanib (Supplemental Figure 8A). Cell viability assays showed synergy in both cell lines at 10-200 nM dinaciclib with $100 \mathrm{nM}$ vandetanib for the TT cells, and at $500 \mathrm{nM}$ and 1,000 nM dinaciclib with 6,000 nM vandetanib (Supplemental Figure 8B). Taken together, these data support the premise that RET overactivation is in part responsible for survival of MTC cells in response to dinaciclib.

CDK7 inhibitor THZ1 is active against MTC cells in vitro. Given the principle effects on RNAP II-associated transcription in the RNA-seq analysis and the reduction in RNAP II activation on Western blot, we sought to target RNAP II function with greater specificity using a different target. CDK7 is a key regulator in the transcription process, by phosphorylating RNAP II to facilitate its release from promoter-proximal pausing. Treatment of both MTC cell lines with THZ1, a specific covalent CDK7 inhibitor, reduced cell viability at low concentrations (Figure 7A). $\mathrm{IC}_{50}$ values were achieved in this assay starting at 24 hours with TT cells and 48 hours for MZ-CRC-1 cells. At 72 hours of exposure, the $\mathrm{IC}_{50}$ for TT cells was $26 \mathrm{nM}$ (95\% CI: 17.8-38.8 $\mathrm{nM})$ and $87 \mathrm{nM}(95 \%$ CI: 69.9-116.8 nM) for MZ-CRC-1 cells. Cell proliferation was also markedly reduced with low concentrations and short exposure times (Figure 7B). $\mathrm{GI}_{50}$ concentrations were also found starting at 24 hours for TT cells and 48 hours for MZ-CRC-1 cells. At 72 hours, GI $_{50}$ was $11.7 \mathrm{nM}$ (95\% CI: 10.4-13.2 $\mathrm{nM}$ ) for TT cells and $34.7 \mathrm{nM}$ (95\% CI: 29.4-41.4 nM) for MZ-CRC-1. Apoptosis was induced with CDK7 inhibition, as evidenced by PARP-1 cleavage in both cell lines at 72 hours (Figure 7C).

We also targeted BRD4, a component in RNAP II transcription regulation, with JQ1. This resulted in decreased cell viability for TT cells but had a very mild effect on MZ-CRC-1 cells (Supplemental Figure 6), consistent with the low levels of BRD4 in the cells when compared with other BRD units, as evaluated in RNA-seq. $\mathrm{IC}_{50}$ was reached at 72 hours for TT cells in this assay, but for MZ-CRC-1 cells it was not reached until 120 hours. At 120 hours of exposure, the $\mathrm{IC}_{50}$ for TT cells was $68.7 \mathrm{nM}$ (95\% CI: 51.2-91.7 nM), while it was $466.8 \mathrm{nM}(95 \%$ CI: $278.8-998.9 \mathrm{nM})$ for MZ-CRC-1.

THZ1 downregulates CDK9 and RET $m R N A$ and protein levels in MTC cells in vitro. We next sought to test whether THZ1 reduced RET and CDK9 mRNA and protein levels in MTC cells. THZ1 treatment had an effect similar to that of dinaciclib on CDK9 protein levels, while CDK7 protein expression remained stable 
A

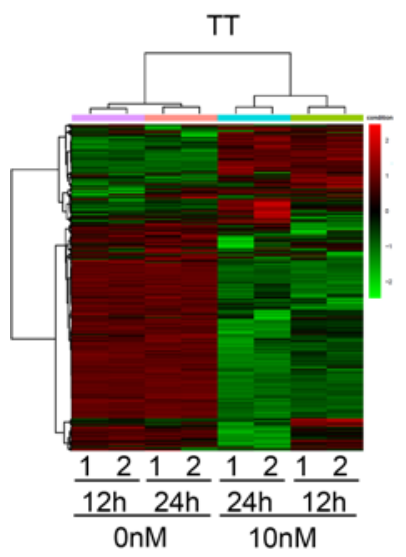

B

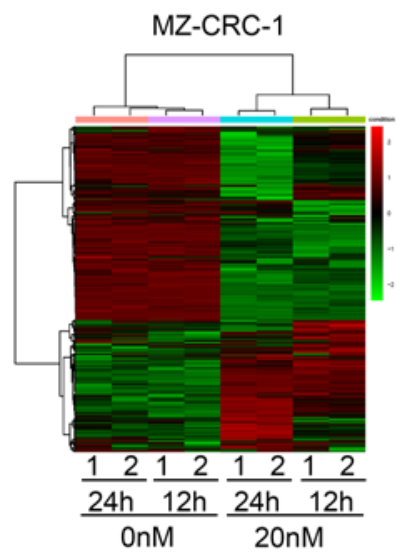

$p<0.001$

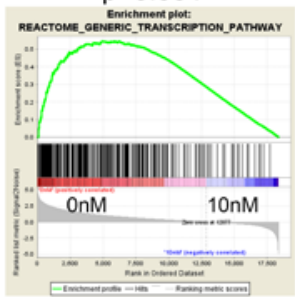

$p<0.001$

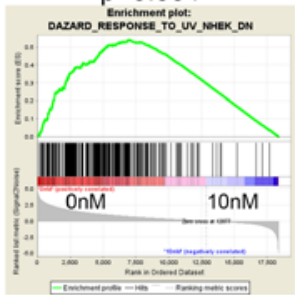

$p<0.001$

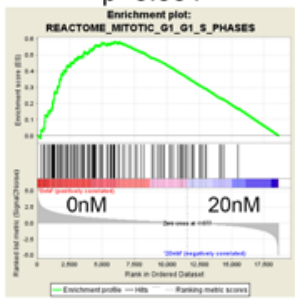

$p<0.001$

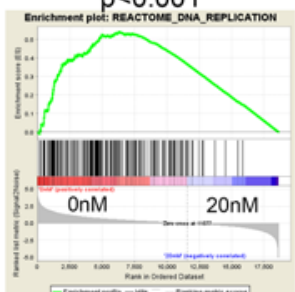

$\mathrm{p}<0.001$

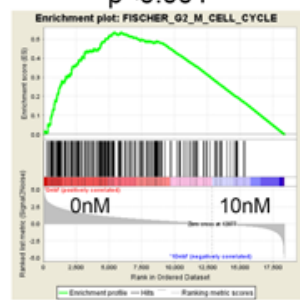

$p<0.001$

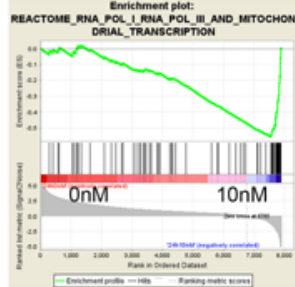

$p<0.001$

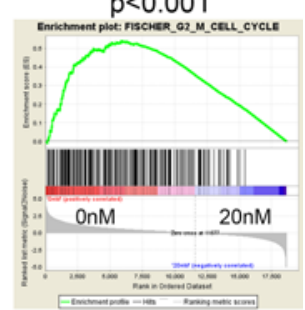

$p<0.001$

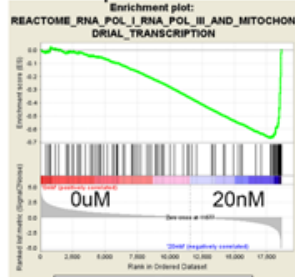

Figure 4. RNA sequencing and gene set enrichment analysis (GSEA) of dinaciclib-treated and non-treated TT and MZ-CRC-1 cells. RNA sequencing was performed on TT (A) and MZ-CRC-1 (B) cells after treatment with dinaciclib or vehicle control for 12 hours and 24 hours. Heatmaps of differentially expressed genes were created, and the genes were compared to curated gene sets; highly differential gene sits are demonstrated. Details of the RNA-seq results can be found in Supplemental Figure 4, and details of enrichment results can be found in Supplemental Tables 1 and 2.

but its function was inhibited (Figure 8A). CDK9 mRNA was significantly reduced at early time points of exposure for both cell lines (Figure 8B). In addition, RET protein and mRNA downregulation was also observed under THZ1 treatment in both cell lines (Figure 9, A and B). RET protein levels decreased with this treatment in both cell lines, and the RET downstream pathway was activated, as noted by the increased ERK phosphorylation with increased THZ1 dose in the surviving cells, similar to the dinaciclib studies (Figure 9A). Both MTC cell lines were treated with THZ1 in the presence or absence of cycloheximide to determine if THZ1 affects CDK9 and/or RET protein stability (Supplemental Figure 7). CDK9 and RET protein levels decreased with THZ1 treatment alone and with cycloheximide treatment alone, and the combined treatment did not further decrease CDK9 or RET protein level. Cyclin D1 levels were reduced by cycloheximide and THZ1.

RET is associated with a superenhancer in TT cells. We performed ChIP-sequencing (ChIP-seq) to identify predicted superenhancers using H3K27Ac and BRD4 antibodies on the TT cell line. Overall, predicted superenhancers were identified in different locations of the genome (Figure 10A), with most enhancers located in either intronic or intergenic regions. Out of 20,158 merged peak regions identified with H3K27Ac immunoprecipitation, 1,008 were classified as superenhancers (top 5\%) (Figure 10B). 
A

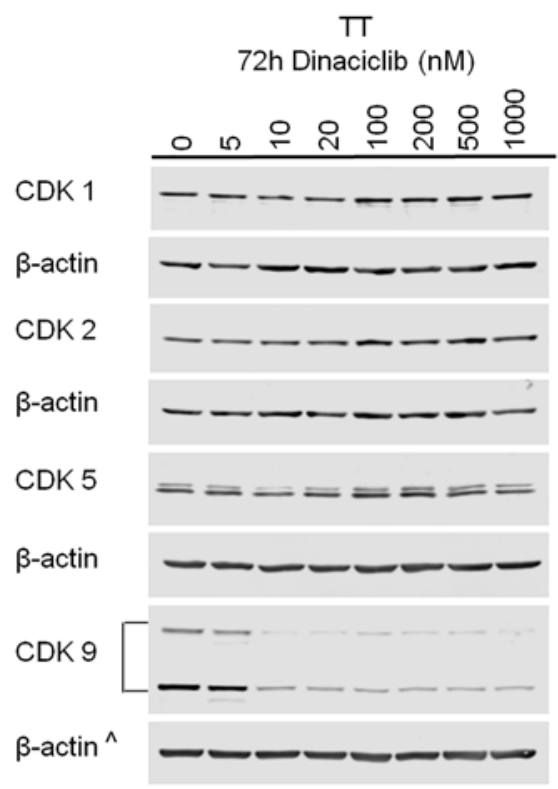

B

TT

72h Dinaciclib (nM)

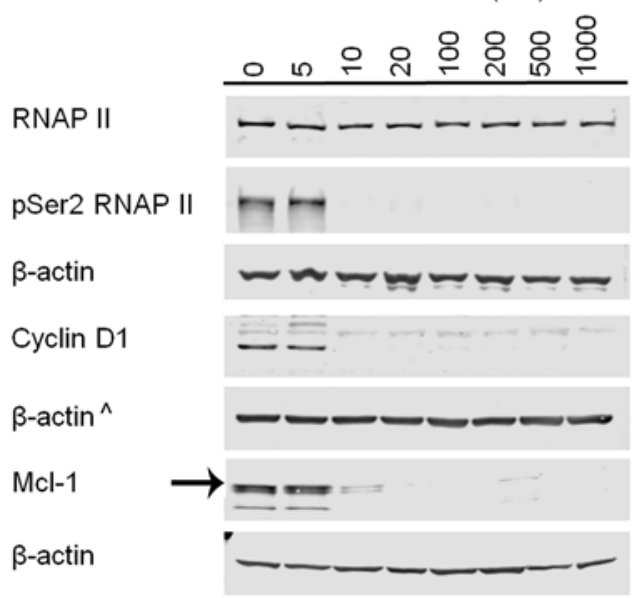

MZ-CRC-1

72h Dinaciclib (nM)

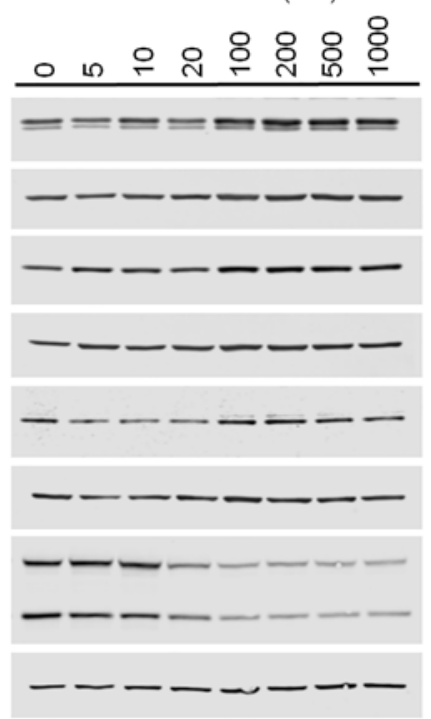

MZ-CRC-1

$72 \mathrm{~h}$ Dinaciclib (nM)

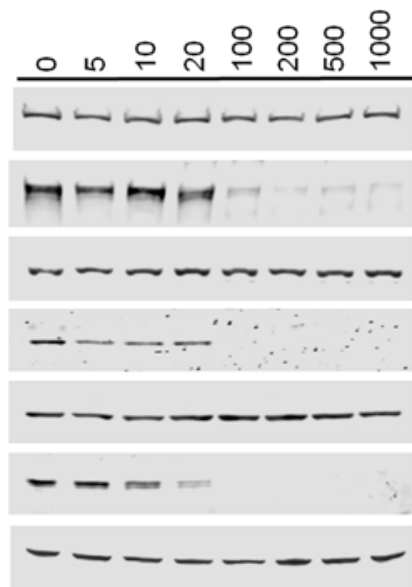

C

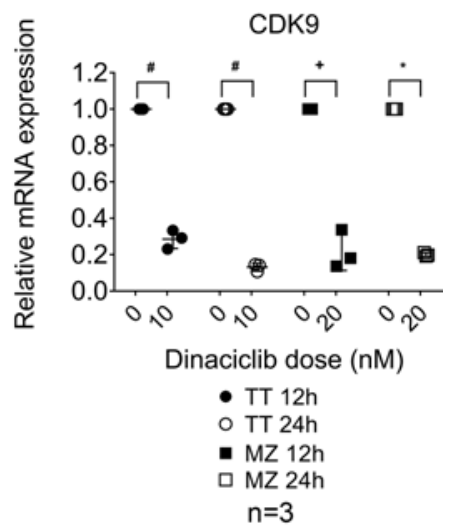

Figure 5. Downregulation of CDK9 protein and mRNA in TT and MZ-CRC-1 cells with dinaciclib treatment. Western blots were performed with protein extracts from TT and MZ-CRC-1 cells treated with dinaciclib or vehicle control for 72 hours. Total CDK9 protein levels were reduced with dinaciclib treatment (A) as well as phosphorylation of RNA polymerase II at Ser2 and downstream proteins cyclin D1 and Mcl-1 (B). Treatment with dinaciclib showed a reduction in CDK9 mRNA (C). RT-qPCR data were normalized to the $0 \mathrm{nM}$ (vehicle control) samples and represented as relative expression. Each biological replicate was performed in duplicate, and the error bars represent the standard deviation. One-way ANOVA tests were performed for the $\Delta C t$ values. ${ }^{*} P<$ $0.001,{ }^{\sharp} P<0.01,{ }^{+} P<0.05$. ${ }^{\wedge}$-Actin for CDK9 and cyclin D1 in TT cells is the same because they were blotted on the same membrane.

The BRD4 ChIP-seq identified 1,806 merged peak regions, 90 of which were classified as superenhancers. Of these 90 superenhancers predicted in the BRD4 ChIP data, 62 also had strong H3K27Ac enrichments. A total of 2,441 genes were found to be associated with the superenhancers identified in this study. These genes are associated with different biological processes, mainly cellular and metabolic processes using the PANTHER database (Figure 10C). Further investigation showed that these included cell communication and primary metabolic processes that involve DNA and RNA metabolism (Supplemental Figure 9).

Importantly, this analysis identified a putative superenhancer within the $R E T$ gene body based on our H3K27Ac ChIP-seq data, with an overall ranking of 43 out of the 1,008 superenhancers called (Figure 10, $\mathrm{B}$ and D). We also observed overlapping BRD4 peaks on the RET gene (Figure 10D), that collectively suggest that RET expression is controlled in MTC TT cancer cell lines by superenhancer activity. 
A

TT

72h Dinaciclib (nM)

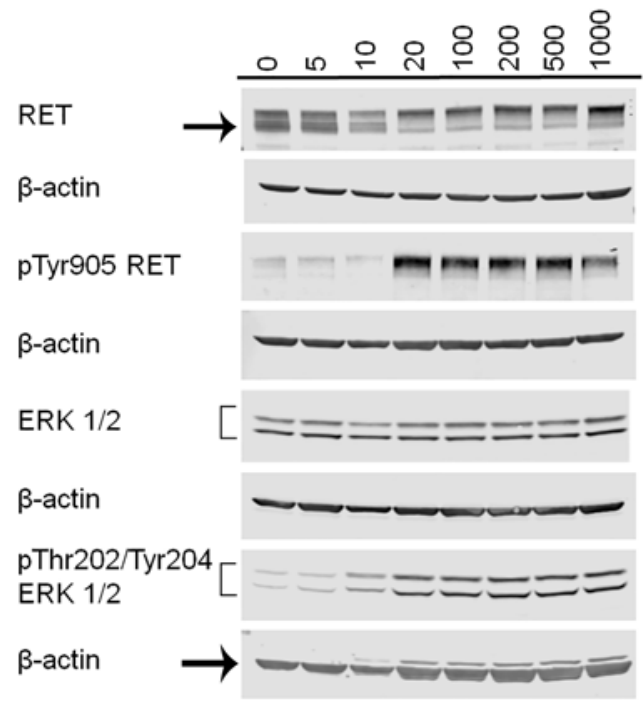

MZ-CRC-1

72h Dinaciclib (nM)

RET

$\beta-\operatorname{actin}^{\wedge}$

pTyr905 RET

$\beta$-actin

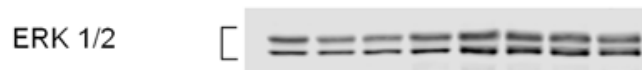

pThr202/Tyr204 ERK 1/2
B

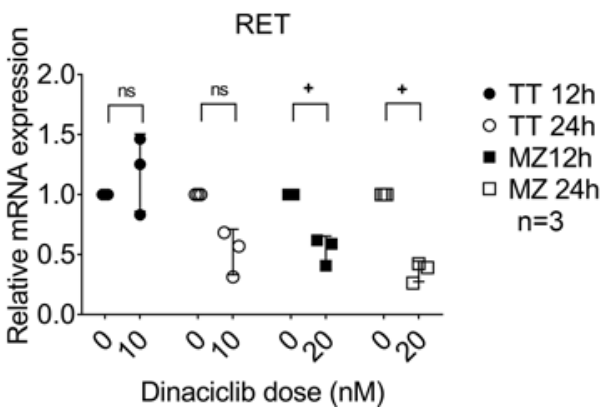

Figure 6. Downregulation of RET protein and mRNA in TT and MZ-CRC-1 cells with dinaciclib treatment. Western blots were performed with protein extracts form TT and MZ-CRC-1 cells treated with dinaciclib or vehicle control for 72 hours. Total RET protein levels were reduced with treatment while phosphorylation of RET and ERK indicate RET pathway activation (A). Treatment with dinaciclib showed a reduction in RET mRNA (B). RT-qPCR data were normalized to the $0 \mathrm{nM}$ (vehicle control) samples and represented as relative expression. Each biological replicate was done in duplicate, and the error bars represent the standard deviation. One-way ANOVA tests were performed for the $\Delta$ Ct values. ${ }^{*} P<0.001,{ }^{\#} P<0.01,{ }^{+} P<0.05$. ns, not significant. ${ }^{\wedge} \beta$-Actin for RET, ERK $1 / 2$, and $p$-Thr202/ Tyr204 ERK $1 / 2$ in MZ-CRC-1 cells is the same because they were blotted on the same membrane.

CDK9 expression in human MTCs. To determine if CDK9 was expressed in human MTCs, DNA from 30 MTC tumors was analyzed on array-comparative genomic hybridization (array-CGH) and it was found that 11 out of 30 cases had $C D K 9$ amplification. Ten cases had 3 copies of $C D K 9$ while 1 case had 4 copies. The rest of the samples had 2 copies of $C D K 9$. Amplification of $C D K 9$ showed a trend towards correlation with lymph node metastasis occurrence in non-RET mutant cases $(P=0.0762)$ (Supplemental Table 11). To validate these data and evaluate CDK9 protein expression, we stained 2 additional cohorts of MTC samples: 1 from OSU and 1 from the University of Michigan. From the OSU cohort, 10 out of 14 samples (from 14 patients) for which MTC and normal thyroid areas were available had stronger CDK9 staining in the tumor areas than in the normal thyroid (Supplemental Figure 10A). In addition, we stained 36 more samples (from 3 patients) from this OSU cohort and found that CDK9 was present in all of the cases; however, there were no normal C-cells to compare to due to the rarity in normal thyroid tissues (Supplemental Figure 10B). The validation cohort from the University of Michigan consisted of 65 MTC tumor microarray samples, which were stained for CDK9 and found to be positive (Supplemental Figure 10C). 
A

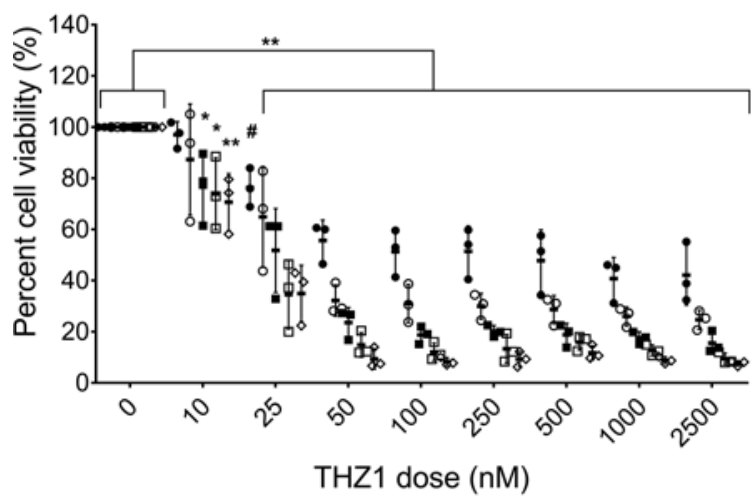

B

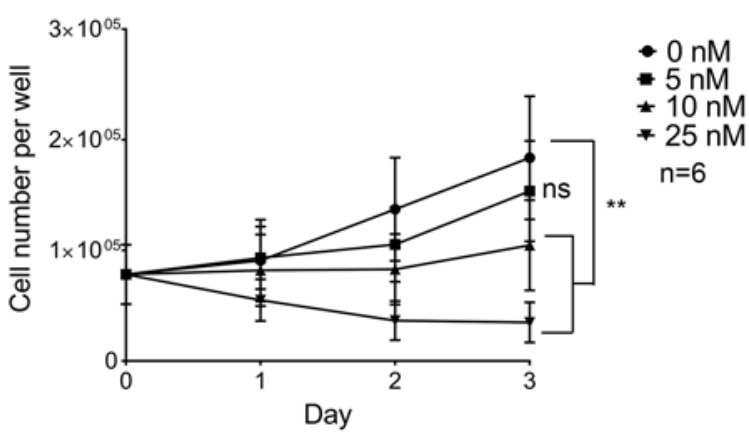

C

$\pi$

$72 \mathrm{~h}$ THZ1 (nM)

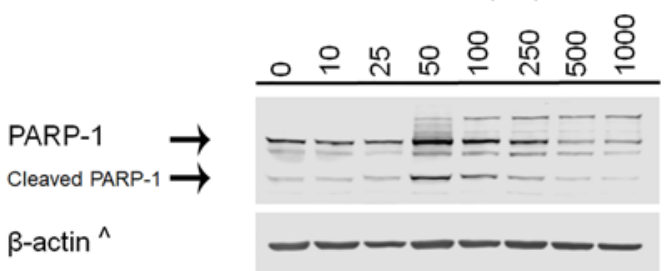

MZ-CRC-1

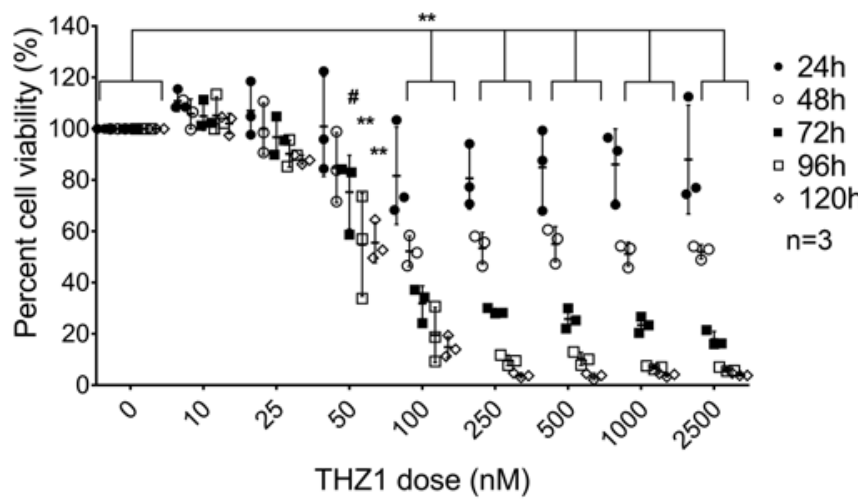

MZ-CRC-1

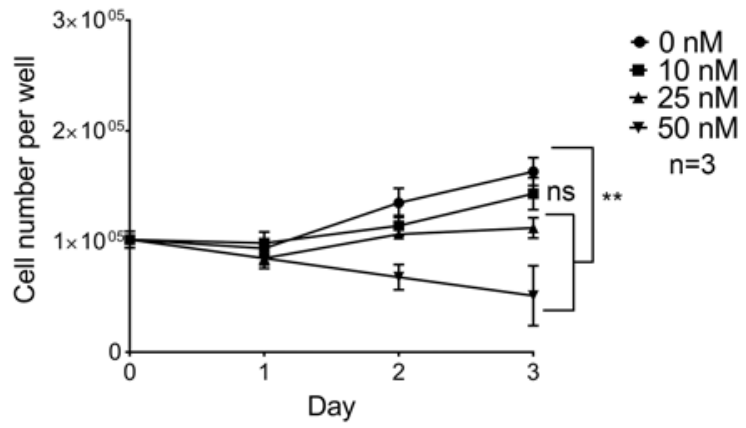

MZ-CRC-1

72h THZ1 (nM)

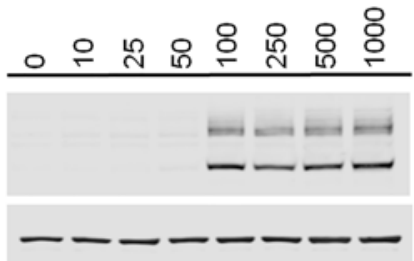

Figure 7. TT and MZ-CRC-1 cell metabolic activity and proliferation after THZ1 treatment. Cell viability assays (A) and cell counting assays (B) were performed with TT and MZ-CRC-1 cells treated with THZ1 or vehicle control. Data were normalized to the $0 \mathrm{nM}$ (vehicle control) wells and represented as percentages. Each biological replicate was performed in triplicate (cell viability assays) or duplicate (cell counting assays), and the error bars represent the standard deviation. Two-way ANOVA tests followed by Holm's procedure were done on both types of assays. In the case of cell counting assays, log -transformed data were used for statistical analysis. (C) Western blots were performed with protein extracts from TT and MZ-CRC-1 cells treated with vehicle control or THZ1 for 72 hours. PARP-1 cleavage was used as a marker of apoptosis and was observed in the treated cells versus vehicle control. ${ }^{* *} P<0.0001$, ${ }^{*} P<0.001,{ }^{\#} P<0.01$. ns, not significant. ${ }^{\wedge} \beta$-Actin for PARP-1 in this figure and for ERK $1 / 2$ and $p$-Thr202/Tyr204 ERK $1 / 2$ in Figure 9 in MZ-CRC-1 cells is the same because they were blotted on the same membrane.

\section{Discussion}

The CDK/RB pathway is a logical potential target for therapy in MTC given the existent data in murine models that show MTC development after loss of $R b$ and negative regulators of this pathway such as 18 and p27, and increased MTC aggressiveness with loss of p18 and p27 in Ret-mutant mice (15-19). Human data further support the importance of this pathway in MTC, since LOH occurs in loci of several RB pathway genes, such as p18 and E2F2, and low RB expression in human MTC is associated with decreased patient survival $(15,21)$. However, targeting this pathway in vitro with a CDK4/6 specific inhibitor (palbociclib) had only modest effects on cell proliferation and was not cytotoxic, even at high doses, and the loss of RB suggests that this may not be a logical approach (Supplemental Figure 1). Similar results have been published by others using similar cell lines suppressing p25 overexpression in a CDK5-driven model (20). 
A

TT

72h THZ1 (nM)

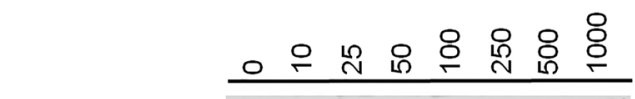

CDK 7

$\beta$-actin

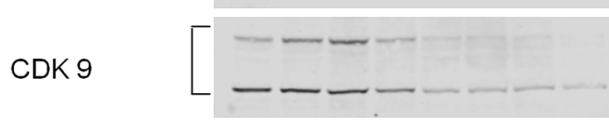

RNAP ॥

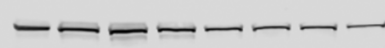

pSer5 RNAP II

$\beta$-actin

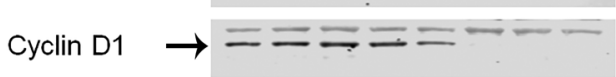

$\beta$-actin ^

Mcl-1

$\beta$-actin

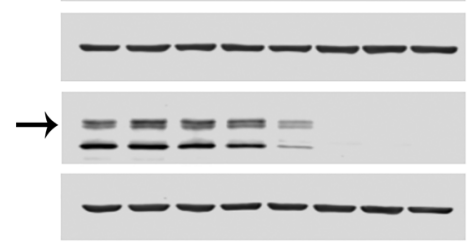

CDK 7

$\beta-\operatorname{actin}^{\wedge \wedge}$

CDK 9

$\beta$-actin

RNAP II

pSer5 RNAP ॥

$\beta$-actin

Cyclin D1

$\beta$-actin

$\mathrm{Mcl}-1$

$\beta$-actin
MZ-CRC-1

72h THZ1 (nM)

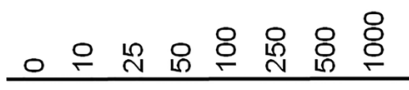

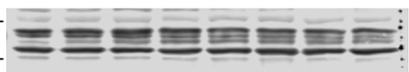
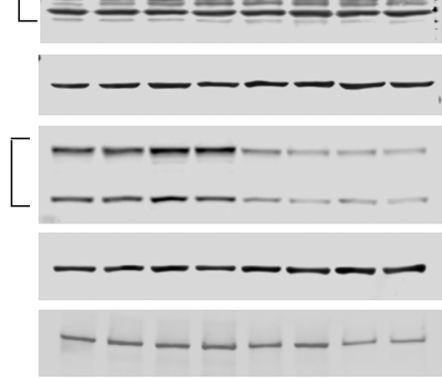

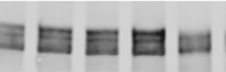
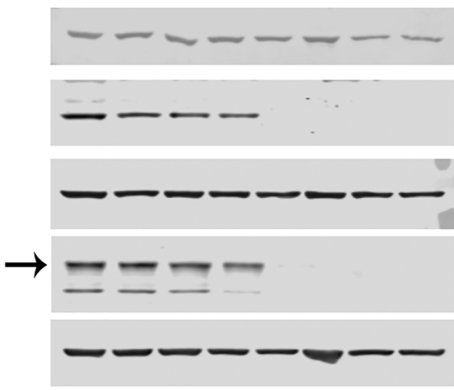

B

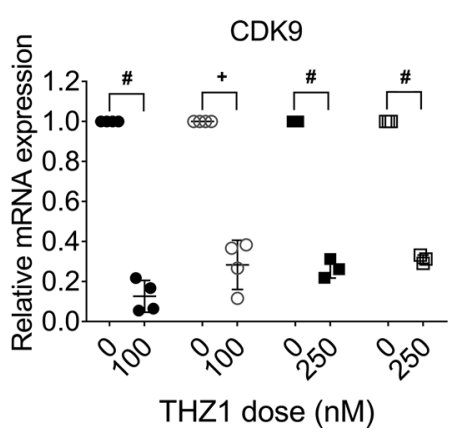

- TT $12 \mathrm{~h} n=4$

- TT 24h $n=4$

- MZ-CRC-1 $12 \mathrm{~h} \mathrm{n}=3$

$\square \mathrm{MZ}-\mathrm{CRC}-12 \mathrm{~h} \mathrm{n}=3$

Figure 8. Downregulation of CDK9 protein and mRNA in TT and MZ-CRC-1 cells with THZ1 treatment. Western blots performed with protein extracts from TT and MZ-CRC-1 cells treated with vehicle control or THZ1 for 72 hours. Total CDK9 protein levels were reduced with THZ1 treatment as well as phosphorylation of RNA polymerase II (RNAP II) at Ser5 and downstream proteins cyclin D1 and Mcl-1, with no change in CDK7 levels (A). Treatment with THZ1 reduced CDK9 mRNA levels (B). RT-qPCR data were normalized to the $0 \mathrm{nM}$ (vehicle control) samples and are represented as relative expression. Each biological replicate was done in duplicate, and the error bars represent the standard deviation. One-way ANOVA tests were performed on the $\triangle \mathrm{Ct}$ values. ${ }^{\#} P<0.01,{ }^{+} P<0.05$. ${ }^{\wedge}$-Actin for cyclin D1 in this figure and for $p$-Thr202/Tyr204 ERK $1 / 2$ in Figure 9 in TT cells is the same because they were blotted on the same membrane. ${ }^{\wedge} \beta$-Actin for CDK7 in this figure and for RET in Figure 9 in MZ-CRC-1 cells is the same because they were blotted on the same membrane. Nu-PAGE 3\%-8\% Bis-Tris gel was used for RNAP II and pSer5 RNAP II in MZ-CRC-1.

However, treatment of both MTC cell lines with the CDK1/2/5/9 inhibitor dinaciclib resulted in significant reductions in cell viability and cell number (Figure 1), and enhanced apoptosis (Figure 3). Interestingly, dinaciclib was similarly potent against MTC cells that were selected for resistance to vandetanib (Figure 2). Thus, we opted to study the mechanism of action.

In an unbiased approach using RNA-seq at doses close to the $\mathrm{IC}_{50}$ of dinaciclib (Figure 4 and Supplemental Tables 1 and 2), we identified a global reduction in most mRNAs that correlated with a primary downregulation of genes involved in RNAP II-mediated transcription, yet RNAP I, RNAP III, and mitochondrial polymerase-related transcripts were upregulated. Specifically, there was a reduction in multiple $M E D$ and $Z N F$ transcripts, which are important regulators of RNAP II and superenhancer functions. Other top gene sets affected were involved in cell cycle, cell division, and DNA replication and repair, as might be 
A

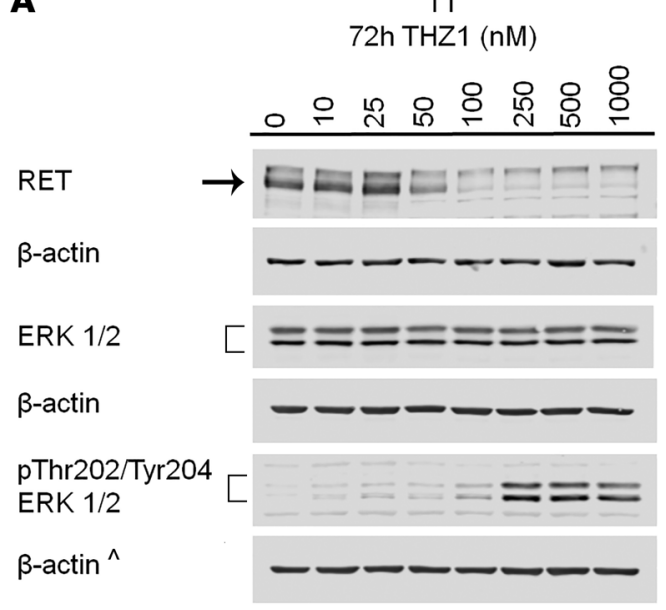

MZ-CRC-1 72h THZ1 (nM)
RET

$\beta-\operatorname{actin}^{\wedge \wedge}$

ERK $1 / 2$

pThr202/Tyr204 ERK $1 / 2$

$\beta$-actin ${ }^{\wedge \wedge \wedge}$

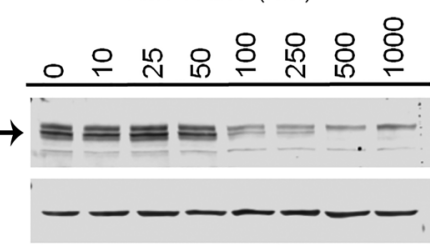

ㄷ=ニニニニニ

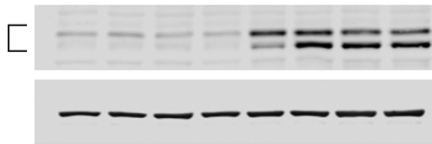

B

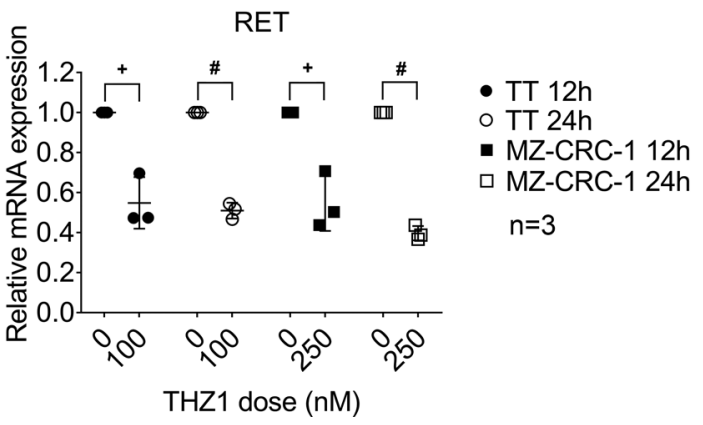

Figure 9. Downregulation of RET protein and mRNA in TT and MZ-CRC-1 cells with THZ1 treatment. Total RET protein levels were reduced with THZ1 treatment while phosphorylation of RET and ERK indicate RET pathway inactivation at lower doses but activation at higher doses in the surviving cells (A). Treatment with THZ1 reduced RET mRNA levels (B). RT-qPCR data were normalized to the $0 \mathrm{nM}$ (vehicle control) samples and represented as relative expression. Each biological replicate was performed in duplicate, and the error bars represent the standard deviation. One-way ANOVA tests were performed on the $\Delta$ Ct values. ${ }^{\#} P<0.01,{ }^{+} P<0.05$. ${ }^{\wedge} \beta$-Actin for $p$-Thr202/Tyr204 ERK $1 / 2$ in this figure and for cyclin D1 in Figure 8 in TT cells is the same because they were blotted on the same membrane. ${ }^{\wedge} \beta$-Actin for RET in this figure and for CDK7 in Figure 8 in MZ-CRC- 1 cells is the same because they were blotted on the same membrane. ${ }^{\wedge \wedge} \beta$-Actin for ERK $1 / 2$ and $p-T h r 202 / T y r 204$ ERK $1 / 2$ in this figure and for PARP-1 in Figure 7 in MZ-CRC- 1 cells is the same because they were blotted on the same membrane.

predicted by the known dinaciclib targets. In addition, upregulation of HIST transcripts in both cell lines under treatment is suggestive of chromatin remodeling, which is a factor regulating gene expression. The accumulation of histones might be due to defective degradation given by downregulation of $S L B P$, a gene whose transcript is downregulated in our RNA-seq results and which plays a key role in regulating histone mRNA metabolism (52), but further experiments need to be performed to test this hypothesis.

As a CDK inhibitor, dinaciclib inhibits the kinase function of its target CDKs. However, CDK9 mRNA and protein levels are unexpectedly reduced by exposure to low concentrations, while the protein levels of other targets were stable (Figure 5). Additionally, downstream targets of CDK9 were inhibited. Interestingly, the loss of CDK9 mRNA and protein occurred at short exposure times and low doses. Importantly, dinaciclib did not alter CDK9 protein stability or induce proteasome-mediated degradation of CDK9 (Supplemental Figure 5). These data are consistent with a possible role for dinaciclib in CDK9 gene transcription or RNA processing in addition to the kinase inhibitory effect, consistent with a more complex mode of action in these cells. The fact that RB loss is seen in human MTCs (21) could suggest that CDK2 might not be the primary target of dinaciclib in these cells that express low levels of wild-type RB (RNA-seq and data not shown).

The RNA-seq and confirmatory RT-PCR studies of TT and MZ-CRC-1 cells treated with dinaciclib also identified downregulation of RET transcript, the key oncogenic driver of MTC and the neuroendocrine tumor syndrome MEN2. It was also interesting that the surviving cells at higher doses of dinaciclib demonstrated an increase in RET activity and an increase in the upper band of the RET doublet and p-RET levels despite the reduction in mRNA expression, consistent with enhanced activation of RET in 

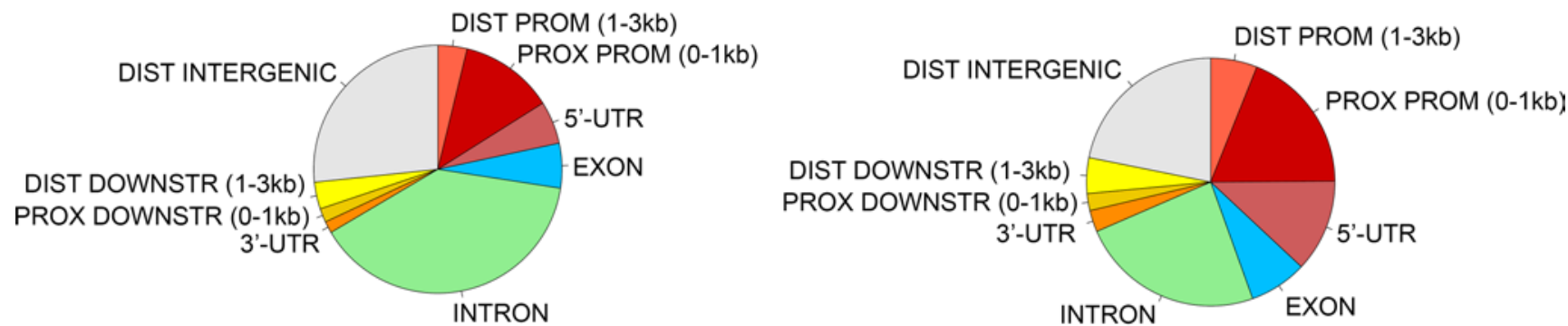

$\mathbf{B}$

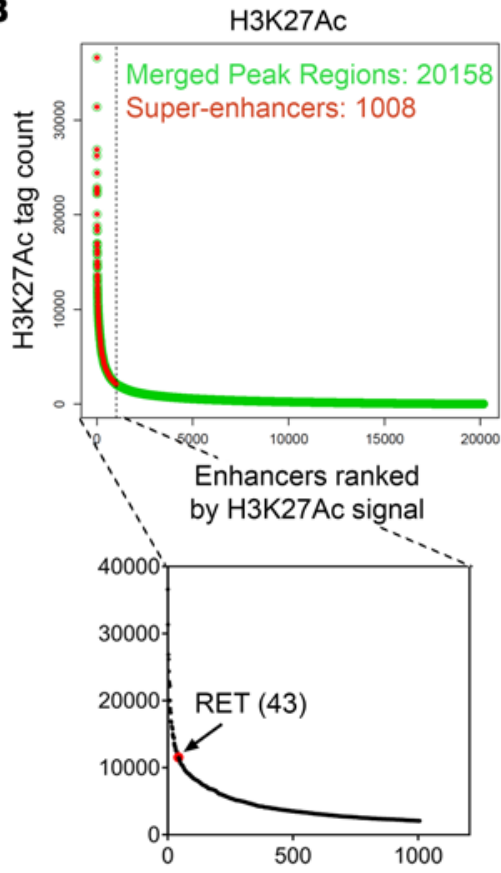

C

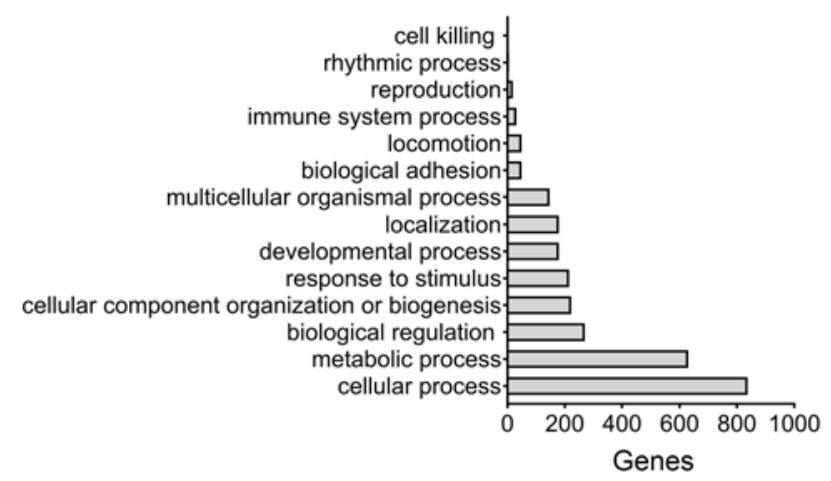

D

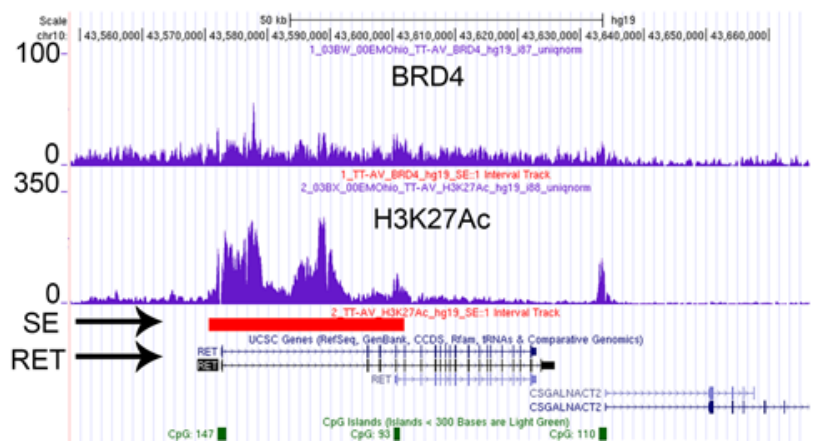

Figure 10. Identification of RET-associated superenhancer in TT cells. ChIP sequencing (ChIP-seq) done for H3K27Ac and BRD4 identified enhancers in different genomic regions of the TT cell genome (A). A total of 1,008 superenhancers were identified with the H3K27Ac ChIP-seq (B). The superenhancer associated genes are distributed into biological processes that are involved in metabolic and cellular processes (C). RET was associated with a superenhancer on the H3K27Ac ChIP-seq (D).

the surviving cell population (Figure 6). The precise mechanism for this further activation could reflect enhanced protein homo- or heterodimerization, as has been described for other receptor tyrosine kinases (53). Nonetheless, this reactivation of RET kinase activity allows potential for synergy between dinaciclib and vandetanib in association with blockade of this reactivation (Supplemental Figure 8).

While the data pointed toward a possible transcriptional primary mechanism, it is recognized that dinaciclib targets several CDKs and the protein changes in RET were complex; thus, we evaluated other compounds that inhibit RNAP II function through other mechanisms with greater specificity. Treatment with the CDK7 inhibitor THZ1 mimicked the results seen with dinaciclib in terms of reduced cell viability and proliferation, apoptosis, CDK9 and RET mRNA and protein reduction, with a more pronounced effect on RET protein levels, and RET downstream pathway reactivation (Figures 7-9). In this case, the reduction of RET protein was more pronounced, likely related to the greater level and specificity of the inhibitor. BRD4 inhibition using JQ1 also reduced cell viability in TT cells but had only a modest effect on MZ-CRC-1 cells (Supplemental Figure 6). The reduced potency with JQ1 in comparison with dinaciclib or THZ1 may 
be attributable to redundant mechanisms present in the cell that help facilitate CDK9 recruitment to gene promoters, such as CDK8 and NF- $\mathrm{kB}$ or other bromodomain proteins in addition to BRD4. Specifically, the RNA-seq data demonstrate that BRD2 is the predominant BRD transcript in these cells. In addition, it has been reported that BRD4 inhibition through JQ1 may be less potent in downregulating RNAP II phosphorylation than agents that reduce BRD4 protein stability (54). Finally, the relative resistance in the MZ-CRC-1 cell line versus TT could also be explained by the low expression of MYC in the MZ-CRC-1 cells compared with TT. JQ1 has been shown to be most effective in $M Y C$-dependent cancer cells (55); thus, if the effect of BRD4 inhibition occurs in part through MYC in the MTC cells, then this also could be a plausible explanation. Finally, it is notable that the MZ-CRC1 cells, while sensitive to both dinaciclib and THZ1, were more resistant than TT cells to these compounds as well. Further studies are required to determine if MYC expression predicts sensitivity to BRD4 and other transcriptionally targeted inhibitors in MTC.

It is of interest that the reduction of CDK9 and RET levels occurred while a number of other proteins remained stable, suggesting some specificity to this effect (Figures 5, 6, 8, and 9). This does not appear to be related to protein translation or stability for CDK9 and occurred at low doses and early time points for both transcripts (Supplemental Figures 5 and 7). The data suggest a primary effect either on specific gene transcription or mRNA stability. The former was implied by the RNA-seq data, leading to the hypothesis that one or both may contain a superenhancer in these cells, which was suggested in silico using the SuperEnhancer Archive (56). ChIP-seq experiments revealed a superenhancer associated with RET (Figure 10), making this the likely mechanism of action by which dinaciclib and THZ1 regulate RET. Interestingly, $C D K 9$ was not associated with a superenhancer on ChIP-seq in the MTC cells and further studies are ongoing to clarify this mechanism. Even though a superenhancer was not found to be linked to $C D K 9$, the data show this protein to be of potential importance for MTC. Array-CGH in an MTC cohort showed CDK9 amplification in 11 of 30 cases (36\%), suggesting a role for this transcription regulator in MTC. It was not possible to test for associations between specific RET or RAS mutations in sporadic or inherited MTCs and CDK9 expression levels due to the relatively high level of expression in all tested samples (suggesting little functional difference) and the lack of routine somatic results on a large enough number of the samples.

In summary, we have identified nanomolar-range sensitivity of MTC cells to the CDK1/2/5/9 inhibitor dinaciclib, and the CDK7 inhibitor, THZ1. The mechanism appears primarily to be transcriptional, particularly for the CDK7 inhibitor, and likely mediated by a previously unreported superenhancer in intron 1 of RET. The continued importance of maintaining or enhancing RET kinase activity to survive the CDK inhibitors also can be utilized for therapeutic synergy with RET kinase inhibition by targeting both transcriptional and signaling "addiction" to the oncogene. This mechanism for RET inhibition for monotherapy and combinatorial strategies may provide a basis for a new approach to inhibiting this pathway in this difficult-to-treat disease.

\section{Methods}

Cell lines and cell culture. Two MTC human cell lines were used for cell-based assays: TT and MZ-CRC-1. The TT cell line contains a pathogenic RET C634W mutation and was derived from a primary MTC tumor from a 77 -year-old white female patient $(57,58)$. These cells were provided by B. Nelkin, John Hopkins, Baltimore, Maryland, USA. MZ-CRC-1 contains the RET M918T pathogenic mutation. This cell line was derived from a malignant pleural effusion of a 43-year-old white female patient with metastatic MTC (58, 59). Both cell lines were cultured in RPMI medium with $20 \%$ fetal bovine serum (FBS), $1 \%$ glutamine, and $1 \%$ nonessential amino acids at $37^{\circ} \mathrm{C}$ and $5 \% \mathrm{CO}_{2}$.

We also used TT and MZ-CRC- 1 cells that had been cultured in medium containing $0.5 \mu \mathrm{M}$ vandetanib for 3 months and selected for vandetanib resistance. Age-matched control cells cultured in regular RPMI medium without vandetanib were used in the appropriate experiments.

Pharmaceutical reagents. The pharmaceutical reagents used are the following: palbociclib (S1116, Selleckchem), inhibitor of CDK4/6 ( $\mathrm{IC}_{50}$ in cell-free assays, $11 \mathrm{nM}$ and $16 \mathrm{nM}$, respectively); dinaciclib (S2768, Selleckchem), inhibitor of CDK1/2/5/9 ( $\mathrm{IC}_{50}$ in cell free assays, 1-4 nM) and CDK4/6 ( $\mathrm{IC}_{50}$ in cell-free assays, $100 \mathrm{nM}$ ); THZ1 (532372, Millipore), inhibitor of CDK7 ( $\mathrm{IC}_{50}$ in cell-free assays, 3.2 $\mathrm{nM}$ ); vandetanib (S1046, Selleckchem), inhibitor of RET and VEGFR; and JQ1 (S7110, Selleckchem), inhibitor of BRD4(1/2) ( $\mathrm{IC}_{50}$ in cell-free assays, $77 \mathrm{nM} / 33 \mathrm{nM}$, respectively). Other pharmaceutical reagents used: cycloheximide (C7698, Sigma-Aldrich), a protein synthesis inhibitor; and bortezomib (S1013, Selleckchem), a proteasome inhibitor. 
Cell viability assays. TT and MZ-CRC- 1 cells were seeded at a density of $2.0 \times 10^{4}$ cells per well in 96-well plates. After 24 hours, the cells were washed once with PBS and incubated in RPMI media at 2\% FBS for an additional 24 hours. The pharmaceutical reagent of interest was added in RPMI media at $2 \%$ FBS. At 24-120 hours of exposure to the drugs, $10 \mu$ of WST-8 (Dojindo Molecular Technologies) was added to each well already containing $100 \mu \mathrm{l}$ of drug/medium and the plates were incubated for 3 hours at $37^{\circ} \mathrm{C}$. The optical density was read at $450 \mathrm{~nm}$ using a spectrophotometer. For assays longer than 72 hours, the treatment or vehicle control was replaced at 72 hours of exposure after aspirating the old treatment or vehicle. The data were plotted using GraphPad software. For combination treatments, dinaciclib was added for 6 hours, after which it was removed, the cells washed with PBS, and vandetanib or RPMI media was added for 72 hours, after which WST- 8 solution was added.

Cell counting assays. TT and MZ-CRC-1 cells were seeded in 12-well plates at a density of $1.0 \times 10^{4}$ cells per well for TT and $1.2 \times 10^{4}$ cells per well for MZ-CRC-1 cells and incubated for 24 hours. After 24 hours, the cells were washed with PBS one time and incubated in RPMI media with 2\% FBS for 24 hours. The next day, the day- 0 control wells were counted using the trypan blue and the Countess Automated Cell Counter (Invitrogen). At this time, the different drugs or vehicle control were added in RPMI media with $2 \%$ FBS to the rest of the plates and incubated at $37^{\circ} \mathrm{C}$. Each day thereafter, the number of cells per well of one plate were counted using trypan blue and the Countess Automated Cell Counter for up to 3, 4 , or 5 days depending on the drug used. For assays longer than 3 days, the treatment or vehicle control was replaced on day 3 after aspirating the old treatment or vehicle. The data were plotted and statistical tests were performed using GraphPad software.

Protein isolation and Western blots. TT and MZ-CRC-1 cells were seeded in 10-cm plates and incubated for 24 hours, then washed with PBS and incubated in 2\% FBS-RPMI medium for another 24 hours. After this time, the cells were treated with the different drugs or vehicle control and incubated at $37^{\circ} \mathrm{C}$. At the desired exposure time, the supernatant was collected into 50-ml tubes, and the cells were scraped and pipetted into the same 50-ml tube containing the corresponding supernatant. After centrifugation at $300 \mathrm{~g}$ for 5 minutes, the supernatant was aspirated and the cell pellet resuspended in $1 \mathrm{ml}$ PBS to wash the cells. The cells were transferred into $1.5-\mathrm{ml}$ tubes and centrifuged again at $500 \mathrm{~g}$ for 5 minutes. Following aspiration of the supernatant, the cells were lysed in mammalian protein extraction reagent (M-PER) (Thermo Fisher Scientific). The cells were incubated in M-PER for 10 minutes on ice to complete lysis and centrifuged at $12,000 \mathrm{~g}$ for 10 minutes. The supernatant was collected and quantified using the Pierce BCA Protein Assay Kit (Thermo Fisher Scientific).

For Western blots, $20 \mu \mathrm{g}$ of protein for each sample was pretreated with $1 \times$ SDS buffer (Invitrogen) and boiled for 5 minutes before loading into NuPAGE 4\%-12\% Bis-Tris gels (Invitrogen) unless otherwise noted in the figure legend. Proteins were separated by electrophoresis at $180 \mathrm{~V}$ for 1.5 hours and transferred to nitrocellulose membranes (Bio-Rad) at $30 \mathrm{~V}$ for 2 hours. Membranes were blocked using 5\% milk in $1 \times$ TBST and incubated overnight at $4^{\circ} \mathrm{C}$ in primary antibodies as noted below. Membranes were washed using $1 \times$ TBST 3 times for 5 minutes each and incubated in near-infrared fluorescent secondary antibody (LI-COR) for 1 hour at room temperature. Membranes were washed again with $1 \times$ TBST as before and the Odyssey CLx Imaging System (LI-COR) was used for signal acquisition. Each membrane was blotted for a loading control.

The primary antibodies were used to detect the following proteins: PARP-1 (1:1,000 dilution, 9542S, Cell Signaling), $\beta$-actin (1:100,000 dilution, A5316, Sigma-Aldrich), CDK1 (1:1,000 dilution, sc-954, Santa Cruz), CDK2 (1:1,000 dilution, sc-163, Santa Cruz), CDK5 (1:1,000 dilution, sc-750, Santa Cruz), CDK9 (1:500 dilution, 2316S, Cell Signaling), CDK7 (1:1,000 dilution, 2090S, Cell Signaling), RNAP II (1:500 dilution, sc-56767, Santa Cruz), p-Ser2 RNAP II (1:500 dilution, 13499S, Cell Signaling), p-Ser5 RNAP II (1:500 dilution, 13523S, Cell Signaling), Mcl-1 (1:500 dilution, 5453S, Cell Signaling), cyclin D1 (1:500 dilution, sc-753, Santa Cruz), RET (1:500 dilution, 3220S, Cell Signaling), p-Tyr905 RET (1:500 dilution, 3221S, Cell Signaling), ERK (1:1,000 dilution, 4695S, Cell Signaling), and p-ERK (1:500 dilution, 9101L, Cell Signaling).

$R N A$ isolation and $R T-q P C R$. The cells were seeded and treated in $10-\mathrm{cm}$ plates as for the protein isolation assays. At the desired exposure time, the supernatant and scraped cells were collected into 50-ml tubes, centrifuged at $300 \mathrm{~g}$ for 5 minutes, the supernatant aspirated, and the pellet washed with $1 \mathrm{ml}$ of PBS and transferred into $1.5-\mathrm{ml}$ tubes. After centrifugation at $500 \mathrm{~g}$ for 5 minutes, the supernatant was aspirated and the pellet resuspended in $1 \mathrm{ml}$ of TRIzol reagent (Invitrogen) to isolate total RNA. RNA was isolated using the protocol provided by Invitrogen for TRIzol reagent and quantified using the NanoDrop Spectrophotometer (Thermo Fisher Scientific). 
To remove contaminating DNA, 400 ng of RNA was treated with DNAse I (Invitrogen) for 15 minutes at room temperature and at $65^{\circ} \mathrm{C}$ for 10 minutes. The RNA was reverse transcribed into cDNA by using MultiScribe reverse transcriptase (Applied Biosystems) following the manufacturer's instructions. The mRNA levels of CDK9 and RET were detected via quantitative PCR (qPCR) using Taqman assays (CDK9: Hs00977896_g1, RET: Hs01120030_m1, Applied Biosystems) and normalized to 18S ribosomal RNA as previously described (60). The data were analyzed using Mx3000P software (Stratagene) and plotted and analyzed using GraphPad.

RNA-seq. After treating the MTC cells with dinaciclib in $10-\mathrm{cm}$ dishes, the RNA was isolated and treated with DNAse I as described above. Poly-A sequencing was performed using Illumina HiSeq 4000 next-generation sequencer at the Genomics Shared Resource at The Ohio State University. Manual library generation for sequencing was performed by poly-A selection. RNA-seq reads were first mapped to the human genome GRCh38 build using TopHat (version 2.0.13) (22). Raw read counts for each gene were quantified by using the featureCounts (version 1.4.6) (23) tool. Then NCBI RefSeq was used for gene annotation. The RNA-seq data have been deposited in NCBI's Gene Expression Omnibus (61) and can be accessed using GEO Series accession number GSE114070.

GSEA and STRING analyses. The differentially expressed transcripts obtained through RNA-seq after treatment with dinaciclib for 12 hours and 24 hours were compared to curated gene sets from online pathway databases, publications in PubMed, and knowledge of domain experts using the GSEA tool (62, 63). The following options were selected or input into the software for analysis: phenotypes analyzed: (a) $0 \mathrm{nM}$, 12 hours, and 24 hours; (b) $10 \mathrm{nM} / 20 \mathrm{nM}, 12$ hours, and 24 hours; gene set database: c2.all.v6.1.symbols [curated]; number of permutations: 1,000; permutation type: gene_set; Chip platform: HTA_2_0.

The results from GSEA are evaluated based on the enrichment score (ES), which represents the extent to which a gene set is overrepresented at the top or bottom of a ranked list of genes of interest. The normalized enrichment score (NES) accounts for the difference in gene set size and in correlations between the gene set and the expression dataset. Other values reflected in the analysis is the nominal $P$ value, which represents the significance of the enrichment score, and the false discovery rate (FDR), which indicates the probability that the results represent a false positive finding $(62,63)$.

Using GSEA, the leading edge analysis of the upregulated pathways in the treated and nontreated cells was analyzed. Sets of genes were common to multiple pathways in leading edge analysis, and the genes in these pathways were then input into the STRING database (64) to determine Gene Ontology biological functions.

ChIP-seq. Cells were fixed with $1 \%$ formaldehyde for 15 minutes, quenched with $0.125 \mathrm{M}$ glycine, flashfrozen, and sent to Active Motif for ChIP-seq analysis. Briefly, to isolate the chromatin, the cells were lysed and homogenized using a Dounce homogenizer followed by sonication to fragment the DNA to an average 300-500 bp in length. The DNA was treated with RNase, proteinase K, and heat to de-crosslink, followed by ethanol precipitation. Samples containing $30 \mu \mathrm{g}$ of chromatin were precleared using protein A agarose beads (Invitrogen), followed by incubation with $4 \mu \mathrm{g}$ of BRD4 and H3K27Ac antibodies to bind DNA regions. The DNA-protein complexes were washed and eluted using SDS buffer. De-crosslinking of the complexes was performed overnight at $65^{\circ} \mathrm{C}$, and the DNA was purified using the phenol-chloroform method and ethanol precipitation. Specific genomic regions binding to BRD4 and H3K27Ac were checked via RT-qPCR with SYBR Green Supermix (Bio-Rad) to confirm success of the ChIP assay.

Input and ChIP DNA were used to create Illumina sequencing libraries after end-polishing, dA-addition, and adaptor ligation. The libraries were quantified and sequenced using Illumina's NextSeq 500, and the BWA algorithm (65) was used to align the reads to the human genome. After removing duplicate reads, the alignments were extended to $300 \mathrm{bp}$ at their $3^{\prime}$ ends. Genomic locations associated with peaks were determined using the MACS algorithm (66) and a cutoff value of $P=1 \times 10^{-5}$. Removal of peaks that are known to be false based on the ENCODE blacklist were removed. Active Motif's analysis program was used to generate peak locations, metrics, and gene annotations based on the signal maps and peak locations obtained from the MACS algorithm. The data from ChIP-seq were deposited in in NCBI's Gene Expression Omnibus (61) under accession number GSE114070.

Superenhancers were determined based on a bioinformatics approach including peak calling, merging neighboring peaks (MACS peaks for which their inner distance is equal or less than $12,500 \mathrm{bp}$ ), and finally identifying the top $5 \%$ merged peak regions superenhancers. Genes associated with superenhancers were determined as any gene that was $25 \mathrm{~kb}$ or closer to a superenhancer region. PANTHER classification system was used to segregate the superenhancer-associated genes into Gene Ontology biological processes (67). 
Array-CGH analysis. CDK9 copy number of $30 \mathrm{MTC}$ cases was obtained through gene-specific sampling of the array-CGH data reported in Ye et al. (68). The data derive from the use of the Agilent oligonucleotide genomic hybridization microarray $244 \mathrm{~K}$ platform. A $\log _{2}$ ratio greater than 0.5 was considered as DNA amplification. As the microarray platform contains only a single probe specifically mapping to the $C D K 9$ gene (Feature Number 213215), we defined amplification based on signal concordance within a region mapping between probes 21877 and $225864(\sim 100,000 \mathrm{bp})$ that includes the SH2DB3C, CDK9, $F P G S$, and ENG genes.

Tissue microarray. Formalin-fixed, paraffin-embedded tissue blocks (FFPE) of 73 MTC cases (41 primary tumors, 1 recurrent case, and 32 metastatic) were obtained from the files of the Department of Pathology, University of Michigan Medical Center, Ann Arbor, Michigan, USA. After pathological review, a tissue microarray was constructed from the most representative area using the methods described by Nocito et al. (69). Each case was represented by three 1-mm-diameter cores, obtained from the most representative, non-necrotic area of the tumor.

\section{Immunohistochemistry}

OSU samples. MTC tissue slides were stained with CDK9 antibody following a previously described protocol by our group using Vector kits (Vector Laboratories) (21). Slides of paraffin-embedded MTC tissue were deparaffinized with xylene and incubated in a sequence of ethanol dilutions (100\%, 95\%, and 70\%). Antigen unmasking was performed by incubation the slides in a citrate-based antigen and microwaving for 5.5 minutes, followed by a 10 -minute incubation in 3\% hydrogen peroxide after cool down to quench endogenous peroxidase activity. Horse serum was used as a blocking agent for 30 minutes at room temperature. CDK9 primary antibody (2316S, Cell Signaling, 1:100 dilution) was used to incubate the slides overnight at $4^{\circ} \mathrm{C}$. The next day, species specific biotinylated secondary antibody was used to incubate the slides for 1 hour at room temperature. Slides were incubated in Streptavidin/peroxidase for 10 minutes followed by diaminobenzidine substrate (DAB Peroxidase Substrate Kit) for 5 minutes and hematoxylin counterstain for 3 minutes. The slides were washed with water, sealed, and quantified using the Automated Quantitative Pathology Imaging System to determine nuclear expression of CDK9. Ten fields of view were quantified per slide, and $\mathrm{H}$ scores were calculated.

University of Michigan samples. Immunostaining was performed on the DAKO Autostainer (DAKO) using DAKO Envision+ and DAB as the chromogen. Briefly, deparaffinized TMA sections were labeled with CDK9 (2316S, Cell Signaling, 1:200 dilution) for 60 minutes at room temperature. High temperature high pH (10 mM Tris $\mathrm{HCl}$ buffer pH 9/1 mM EDTA) epitope retrieval was used prior to staining. Appropriate negative (no primary antibody) and positive controls were stained in parallel with each set of tumors studied.

\section{Statistics}

Cell viability assays. $\mathrm{IC}_{50}$ values were estimated using 4- or 5-parameter logistic regressions. For optical density cell viability data, 2-way ANOVA tests followed by Holm's procedure were used to determine significance of different dose and exposure time combinations for all cell lines and compounds used and to control for multiple comparisons. The optical density over the tested drug concentrations was compared between agematched control and vandetanib-resistant cells at different time points with linear mixed models after using $\log _{2}$ transformation of the data to reduce skewness and variance. These models were used to account for the correlation among observations from the same experiment. In addition, trend analyses were performed to determine if there were significant slope differences with the increase of drug concentrations between agematched controls and vandetanib-resistant cells with dinaciclib treatment for each time point analyzed. For combination treatments, linear mixed models were used to test the interaction of dinaciclib and vandetanib at each of the dose combinations. If the difference in the optical density ([combo - dinaciclib] - [vandetanib - control]) was less than 0 and the $P$ value was significant, then we claimed there is significant synergistic interaction at the tested dose. Holm's procedure was used to control for multiple comparisons.

Cell counting assays. The cell counting data was first $\log _{2}$ transformed before the analysis to reduce skewness and variance. However, for graphics, the raw count was used. Two-way ANOVA followed by Holm's procedure was used to determine the statistical significance of each dose and exposure time combinations on cell number. Trend analyses were performed to determine the difference between each drug treatment concentration and vehicle control ( $0 \mathrm{nM}$ concentration) across the entire duration of the counting experiments with linear mixed-effects models. 
$R T-q P C R$. One-way ANOVA tests were used to compare the $\triangle \mathrm{Ct}$ between the vehicle control and the treated samples. However, for graphics, the mRNA expression of treated samples was normalized to that of vehicle control.

RNA-seq. Genes with raw read counts below 5 for more than $50 \%$ of samples within each condition were first filtered out. Count data were normalized using the trimmed mean of M-values (TMM) method within controls or drug treatments due to global drug inhibition effect. To improve the estimate of gene variability and to identify genes differentially expressed between samples, R package limma with Voom transformation (70) was used for testing differential expression between conditions. The $P$-value cutoffs were determined by controlling the mean number of false positives (71). Heatmaps with hierarchical clustering were generated, and principal component analysis was performed. Other normalization methods were used as well: normalizing across groups with the least fold-changed transcripts and normalizing within groups with the least fold-changed transcripts. Both of these normalization procedures yielded similar results.

Array-CGH. The association between CDK9 amplification and lymph node metastases or distant metastases occurrence was tested using Fisher's exact test.

Study approval. The sample collections were performed with institutional IRB approvals from The Ohio State University, MD Anderson Cancer Center, and the University of Michigan. In all cases, samples were deidentified prior to inclusion in the study as per approved protocols at the 3 institutions.

\section{Author contributions}

The study conception and design is attributed to MDR, AV, MS, and GJC. Data acquisition was performed by AV, MS, CB, GJC, MDR, TJG, and CMK. The data were analyzed and interpreted by AV, MDR, LY, $\mathrm{XZ}$, ASY, and WM. Drafting of the manuscript is attributed to AV and MDR but reviewed by all authors with subsequent modifications to the final draft.

\section{Acknowledgments}

This work was supported by NCI grant P50 CA168505 to M.D. Ringel, NIH cancer center grant P30 CA016058 to The Ohio State University, 5F31CA210556 to A. Valenciaga, and NIH grant P30 CA046592 to the University of Michigan.

Address correspondence to: Matthew D. Ringel, 565 McCampbell Hall; 1581 Dodd Drive, Columbus, Ohio 43210, USA. Phone: 614.685.3333; Email: matthew.ringel@osumc.edu.

1. Enewold L, et al. Rising thyroid cancer incidence in the United States by demographic and tumor characteristics, 1980-2005 Cancer Epidemiol Biomarkers Prev. 2009;18(3):784-791.

2. Cancer Stat Facts: Thyroid Cancer. National Institutes of Health: National Cancer Institute. https://seer.cancer.gov/statfacts/ html/thyro.html. Accessed August 3, 2018.

3. Links TP, Verbeek HH, Hofstra RM, Plukker JT. Endocrine tumours: progressive metastatic medullary thyroid carcinoma: firstand second-line strategies. Eur J Endocrinol. 2015;172(6):R241-R251.

4. Moley JF. Medullary thyroid carcinoma: management of lymph node metastases. J Natl Compr Canc Netw. 2010;8(5):549-556.

5. Modigliani E, et al. Prognostic factors for survival and for biochemical cure in medullary thyroid carcinoma: results in 899 patients. The GETC Study Group. Groupe d'étude des tumeurs à calcitonine. Clin Endocrinol (Oxf). 1998;48(3):265-273.

6. Wells SA, et al. Vandetanib in patients with locally advanced or metastatic medullary thyroid cancer: a randomized, doubleblind phase III trial. J Clin Oncol. 2012;30(2):134-141.

7. Schoffski P, et al. An international, double-blind, randomized, placebo-controlled phase III trial (EXAM) of cabozantinib (XL184) in medullary thyroid carcinoma (MTC) patients (pts) with documented RECIST progression at baseline. J Clin Oncol. 2012; 30:15_suppl;5508.

8. Alonso-Gordoa T, Díez JJ, Durán M, Grande E. Advances in thyroid cancer treatment: latest evidence and clinical potential. Ther Adv Med Oncol. 2015;7(1):22-38.

9. Elisei R, et al. Cabozantinib in progressive medullary thyroid cancer. J Clin Oncol. 2013;31(29):3639-3646

10. Kushchayeva Y, et al. The HIV protease inhibitor nelfinavir down-regulates RET signaling and induces apoptosis in medullary thyroid cancer cells. J Clin Endocrinol Metab. 2014;99(5):E734-E745.

11. Wells SA, et al. Revised American Thyroid Association guidelines for the management of medullary thyroid carcinoma. Thyroid. $2015 ; 25(6): 567-610$.

12. Howell GM, Hodak SP, Yip L. RAS mutations in thyroid cancer. Oncologist. 2013;18(8):926-932.

13. Ciampi R, et al. Evidence of a low prevalence of RAS mutations in a large medullary thyroid cancer series. Thyroid. 2013;23(1):50-57.

14. Boichard A, et al. Somatic RAS mutations occur in a large proportion of sporadic RET-negative medullary thyroid carcinomas and extend to a previously unidentified exon. J Clin Endocrinol Metab. 2012;97(10):E2031-E2035. 
15. Cote GJ, Grubbs EG, Hofmann MC. Thyroid C-cell biology and oncogenic transformation. Recent Results Cancer Res. 2015;204:1-39.

16. Santarpia L, Ye L, Gagel RF. Beyond RET: potential therapeutic approaches for advanced and metastatic medullary thyroid carcinoma. J Intern Med. 2009;266(1):99-113.

17. van Veelen W, et al. Synergistic effect of oncogenic RET and loss of p18 on medullary thyroid carcinoma development. Cancer Res. 2008;68(5):1329-1337.

18. van Veelen W, et al. P18 is a tumor suppressor gene involved in human medullary thyroid carcinoma and pheochromocytoma development. Int J Cancer. 2009;124(2):339-345.

19. Park MS, Rosai J, Nguyen HT, Capodieci P, Cordon-Cardo C, Koff A. p27 and Rb are on overlapping pathways suppressing tumorigenesis in mice. Proc Natl Acad Sci USA. 1999;96(11):6382-6387.

20. Pozo K, et al. The role of Cdk5 in neuroendocrine thyroid cancer. Cancer Cell. 2013;24(4):499-511.

21. Valenciaga A, et al. Reduced retinoblastoma protein expression is associated with decreased patient survival in medullary thyroid cancer. Thyroid. 2017;27(12):1523-1533.

22. Kim D, Pertea G, Trapnell C, Pimentel H, Kelley R, Salzberg SL. TopHat2: accurate alignment of transcriptomes in the presence of insertions, deletions and gene fusions. Genome Biol. 2013;14(4):R36.

23. Liao Y, Smyth GK, Shi W. featureCounts: an efficient general purpose program for assigning sequence reads to genomic features. Bioinformatics. 2014;30(7):923-930.

24. Bhatt DM, et al. Transcript dynamics of proinflammatory genes revealed by sequence analysis of subcellular RNA fractions Cell. 2012;150(2):279-290.

25. Hargreaves DC, Horng T, Medzhitov R. Control of inducible gene expression by signal-dependent transcriptional elongation. Cell. 2009;138(1):129-145.

26. Rahl PB, et al. c-Myc regulates transcriptional pause release. Cell. 2010;141(3):432-445.

27. Buratowski S. Progression through the RNA polymerase II CTD cycle. Mol Cell. 2009;36(4):541-546.

28. Adelman K, Lis JT. Promoter-proximal pausing of RNA polymerase II: emerging roles in metazoans. Nat Rev Genet. 2012;13(10):720-731.

29. Filippakopoulos $\mathrm{P}$, et al. Histone recognition and large-scale structural analysis of the human bromodomain family. Cell. 2012;149(1):214-231.

30. Kanno T, Kanno Y, Siegel RM, Jang MK, Lenardo MJ, Ozato K. Selective recognition of acetylated histones by bromodomain proteins visualized in living cells. Mol Cell. 2004;13(1):33-43.

31. LeRoy G, Rickards B, Flint SJ. The double bromodomain proteins Brd2 and Brd3 couple histone acetylation to transcription. Mol Cell. 2008;30(1):51-60.

32. Christensen CL, et al. Targeting transcriptional addictions in small cell lung cancer with a covalent CDK7 inhibitor. Cancer Cell. 2014;26(6):909-922.

33. Wang Y, et al. CDK7-dependent transcriptional addiction in triple-negative breast cancer. Cell. 2015;163(1):174-186

34. Zhang Z, et al. Preclinical efficacy and molecular mechanism of targeting CDK7-dependent transcriptional addiction in ovarian cancer. Mol Cancer Ther. 2017;16(9):1739-1750.

35. Albert TK, et al. Characterization of molecular and cellular functions of the cyclin-dependent kinase CDK9 using a novel specific inhibitor. Br J Pharmacol. 2014;171(1):55-68.

36. Cho SJ, et al. Xylocydine, a novel Cdk inhibitor, is an effective inducer of apoptosis in hepatocellular carcinoma cells in vitro and in vivo. Cancer Lett. 2010;287(2):196-206

37. Krystof V, Baumli S, Fürst R. Perspective of cyclin-dependent kinase 9 (CDK9) as a drug target. Curr Pharm Des. 2012;18(20):2883-2890.

38. Lam F, et al. Targeting RNA transcription and translation in ovarian cancer cells with pharmacological inhibitor CDKI-73. Oncotarget. 2014;5(17):7691-7704.

39. Lemke J, et al. Selective CDK9 inhibition overcomes TRAIL resistance by concomitant suppression of cFlip and Mcl-1. Cell Death Differ. 2014;21(3):491-502.

40. Yin T, et al. A novel CDK9 inhibitor shows potent antitumor efficacy in preclinical hematologic tumor models. Mol Cancer Ther 2014;13(6):1442-1456.

41. Walsby E, Lazenby M, Pepper C, Burnett AK. The cyclin-dependent kinase inhibitor SNS-032 has single agent activity in AML cells and is highly synergistic with cytarabine. Leukemia. 2011;25(3):411-419.

42. Walsby E, et al. A novel Cdk9 inhibitor preferentially targets tumor cells and synergizes with fludarabine. Oncotarget. 2014;5(2):375-385.

43. Zhu X, et al. Bromodomain and extraterminal protein inhibitor JQ1 suppresses thyroid tumor growth in a mouse model. Clin Cancer Res. 2017;23(2):430-440.

44. Enomoto K, et al. Targeting MYC as a therapeutic intervention for anaplastic thyroid cancer. J Clin Endocrinol Metab. 2017;102(7):2268-2280.

45. Mio C, et al. BET bromodomain inhibitor JQ1 modulates microRNA expression in thyroid cancer cells. Oncol Rep. 2018;39(2):582-588

46. Hnisz D, et al. Super-enhancers in the control of cell identity and disease. Cell. 2013;155(4):934-947.

47. Hnisz D, et al. Convergence of developmental and oncogenic signaling pathways at transcriptional super-enhancers. Mol Cell. 2015;58(2):362-370.

48. Di Micco R, et al. Control of embryonic stem cell identity by BRD4-dependent transcriptional elongation of super-enhancerassociated pluripotency genes. Cell Rep. 2014;9(1):234-247.

49. Kwiatkowski N, et al. Targeting transcription regulation in cancer with a covalent CDK7 inhibitor. Nature. 2014;511(7511):616620 .

50. Lovén J, et al. Selective inhibition of tumor oncogenes by disruption of super-enhancers. Cell. 2013;153(2):320-334

51. Dawson MA, et al. Recurrent mutations, including NPM1c, activate a BRD4-dependent core transcriptional program in acute myeloid leukemia. Leukemia. 2014;28(2):311-320. 
52. Marzluff WF, Koreski KP. Birth and death of histone mRNAs. Trends Genet. 2017;33(10):745-759.

53. Perlman A, Lawsin LM, Kolachana P, Saji M, Moore J, Ringel MD. Angiotensin II regulation of TGF-beta in murine mesangial cells involves both PI3 kinase and MAP kinase. Ann Clin Lab Sci. 2004;34(3):277-286.

54. Winter GE, et al. BET bromodomain proteins function as master transcription elongation factors independent of CDK9 recruitment. Mol Cell. 2017;67(1):5-18.e19.

55. Fowler T, et al. Regulation of MYC expression and differential JQ1 sensitivity in cancer cells. PLoS One. 2014;9(1):e87003.

56. Wei Y, et al. SEA: a super-enhancer archive. Nucleic Acids Res. 2016;44(D1):D172-D179.

57. Zabel M, Grzeszkowiak J. Characterisation of thyroid medullary carcinoma TT cell line. Histology and Histopathology. 1997;12(1):283-289.

58. Cooley LD, Elder FF, Knuth A, Gagel RF. Cytogenetic characterization of three human and three rat medullary thyroid carcinoma cell lines. Cancer Genet Cytogenet. 1995;80(2):138-149.

59. Knuth A, Gabbert H, Moll R, et al. Markers in medullary thyroid cancer established in tissue culture. Proceedings of the American Association for Cancer Research Annual Meeting. 1987;28.

60. Ringel MD, et al. Overexpression and overactivation of Akt in thyroid carcinoma. Cancer Res. 2001;61(16):6105-6111.

61. Edgar R, Domrachev M, Lash AE. Gene Expression Omnibus: NCBI gene expression and hybridization array data repository Nucleic Acids Res. 2002;30(1):207-210.

62. Subramanian A, et al. Gene set enrichment analysis: a knowledge-based approach for interpreting genome-wide expression profiles. Proc Natl Acad Sci USA. 2005;102(43):15545-15550.

63. Mootha VK, et al. PGC-1alpha-responsive genes involved in oxidative phosphorylation are coordinately downregulated in human diabetes. Nat Genet. 2003;34(3):267-273.

64. Szklarczyk D, et al. The STRING database in 2017: quality-controlled protein-protein association networks, made broadly accessible. Nucleic Acids Res. 2017;45(D1):D362-D368.

65. Li H, Durbin R. Fast and accurate short read alignment with Burrows-Wheeler transform. Bioinformatics. 2009;25(14):1754-1760

66. Zhang Y, et al. Model-based analysis of ChIP-Seq (MACS). Genome Biol. 2008;9(9):R137.

67. $\mathrm{Mi} \mathrm{H}$, et al. PANTHER version 11: expanded annotation data from Gene Ontology and Reactome pathways, and data analysis tool enhancements. Nucleic Acids Res. 2017;45(D1):D183-D189.

68. Ye L, Santarpia L, Cote GJ, El-Naggar AK, Gagel RF. High resolution array-comparative genomic hybridization profiling reveals deoxyribonucleic acid copy number alterations associated with medullary thyroid carcinoma. J Clin Endocrinol Metab. 2008;93(11):4367-4372.

69. Nocito A, Kononen J, Kallioniemi OP, Sauter G. Tissue microarrays (TMAs) for high-throughput molecular pathology research. Int J Cancer. 2001;94(1):1-5.

70. Law CW, Chen Y, Shi W, Smyth GK. voom: Precision weights unlock linear model analysis tools for RNA-seq read counts. Genome Biol. 2014;15(2):R29.

71. Gordon A, Glazko G, Qiu X, Yakovlev A. Control of the Mean Number of False Discoveries, Bonferroni and Stability of Multiple Testing. Cornell University Library. https://arxiv.org/abs/0709.0366. Accessed August 3, 2018 